\title{
Quasivarieties of pseudocomplemented semilattices
}

\author{
by
}

M. E. A d a ms (New Paltz, N.Y.), W. Dzi o bi a k (Toruń), Matthew Gould (Nashville, Tenn.) and Jürg S ch mid (Bern)

\begin{abstract}
Two properties of the lattice of quasivarieties of pseudocomplemented semilattices are established, namely, in the quasivariety generated by the 3-element chain, there is a sublattice freely generated by $\omega$ elements and there are $2^{\omega}$ quasivarieties.
\end{abstract}

1. Introduction. For background material on quasivarieties, the reader is referred to Burris and Sankappanavar [5] or Mal'cev [10]. The following definition of a quasivariety is but one of several equivalent formulations.

Given a class $\mathbf{K}$ of similar algebras, $\mathbf{I}(\mathbf{K})$ and $\mathbf{H}(\mathbf{K})$ respectively denote the classes of all isomorphic and homomorphic images of algebras in $\mathbf{K}, \mathbf{S}(\mathbf{K})$ denotes the class of all subalgebras of algebras in $\mathbf{K}$, and the classes $\mathbf{P}(\mathbf{K})$ and $\mathbf{P}_{\mathbf{u}}(\mathbf{K})$ consist respectively of all direct products and all ultraproducts of members of $\mathbf{K}$. (The direct product of an empty set of algebras, the trivial algebra, is included in $\mathbf{P}(\mathbf{K})$.) If $\mathbf{K} \supseteq \mathbf{I}(\mathbf{K}) \cup \mathbf{S}(\mathbf{K}) \cup \mathbf{P}(\mathbf{K}) \cup \mathbf{P}_{\mathbf{u}}(\mathbf{K})$ then $\mathbf{K}$ is said to be a quasivariety, while if $\mathbf{K} \supseteq \mathbf{H}(\mathbf{K}) \cup \mathbf{S}(\mathbf{K}) \cup \mathbf{P}(\mathbf{K})$ then $\mathbf{K}$ is a variety. (Every variety is a quasivariety.) The quasivariety generated by $\mathbf{K}$ (the least quasivariety containing $\mathbf{K})$ is denoted by $Q(\mathbf{K})$, and the variety generated by $\mathbf{K}$ is denoted by $V(\mathbf{K})$. In general, $Q(\mathbf{K})=\mathbf{I S P P}_{\mathbf{u}}(\mathbf{K})$ (and $V(\mathbf{K})=\mathbf{H S P}(\mathbf{K})$ ), but when $\mathbf{K}$ and its members are finite, $Q(\mathbf{K})=$ $\operatorname{ISP}(\mathbf{K})$. If $\mathbf{K}$ is finite, say $\mathbf{K}=\left\{A_{1}, \ldots, A_{n}\right\}$, then $Q(\mathbf{K})$ will be denoted by $Q\left(A_{1}, \ldots, A_{n}\right)$, and likewise for $V(\mathbf{K})$.

The quasivarieties contained in a given quasivariety constitute a lattice under the inclusion ordering: the meet of two such is their intersection, and the join is the quasivariety generated by their union. The cardinality of the lattice is at most $2^{\omega}$ if the algebras are of finite type.

Interest in the investigation of lattices of quasivarieties is directly or indirectly devoted to a problem of Mal'cev [9], who asked for a description of all lattices that can be represented isomorphically as lattices of quasivarieties.

1991 Mathematics Subject Classification: Primary 06A12, 08C15. 
The present paper provides an example of a lattice of quasivarieties with an intricate lattice structure demonstrating the complexity of Mal'cev's problem. Amongst those papers referenced here, other examples of this kind are given in [1], [3], [4], [6], [12], [16], and [17].

A pseudocomplemented semilattice is an algebra $(S ; \wedge, *, 0,1)$ comprised of a semilattice $(S ; \wedge)$ with a least element 0 , a greatest element 1 , and a unary operation $*$ such that, for all $s, t \in S, s \wedge t=0$ if and only if $t \leq$ $s^{*}$. Background material on pseudocomplemented semilattices and related topics is to be found in Grätzer [7] and the papers cited below.

The pseudocomplemented semilattice $\widehat{B}$ obtained by adjoining a new greatest element to a Boolean algebra $B$ is of interest because Jones [8] proved that those pseudocomplemented semilattices of the form $\widehat{B}$ are precisely the subdirectly irreducible ones. Letting $B_{m}(m<\omega)$ denote the finite Boolean algebra with $m$ atoms, we obtain a strictly increasing sequence $\left(Q\left(\widehat{B}_{m}\right): m<\omega\right)$ of quasivarieties investigated in [13]. The only non-trivial variety of pseudocomplemented semilattices is $Q\left(\widehat{B}_{0}\right)$ (proved by Jones [8]), which is precisely the class of all Boolean algebras. Thus, $m=0$ is the only case in which $Q\left(\widehat{B}_{m}\right)=V\left(\widehat{B}_{m}\right)$. The gap between $Q\left(\widehat{B}_{1}\right)$ and $V\left(\widehat{B}_{1}\right)$ is particularly striking because $V\left(\widehat{B}_{1}\right)$ is the entire class of pseudocomplemented semilattices (proved in Jones [8]; this and the aforementioned results of Jones are also proved in Sankappanavar [11]).

Our goal is to prove the following result.

THEOREM 1.1. The lattice of all subquasivarieties of the quasivariety $Q\left(\widehat{B}_{1}\right)$ generated by the 3 -element pseudocomplemented semilattice $\widehat{B}_{1}$ has a sublattice freely generated by $\omega$ elements (hence, satisfies no non-trivial lattice identity) and is of cardinality $2^{\omega}$.

To put this theorem in perspective, it should be noted that the lattice of quasivarieties of pseudocomplemented distributive lattices has a sublattice freely generated by $\omega$ elements (Tropin [16]; see also [6]) and has cardinality $2^{\omega}$ (independently proved in [1] and Wroński [17]). Moreover, the existence of $2^{\omega}$ quasivarieties of pseudocomplemented semilattices was established in [4]; by applying a criterion of [13] it can be shown that the quasivarieties exhibited in [4] lie in $Q\left(\widehat{B}_{3}\right)$. Additional related results can be found in [13] and [14]. We remark that, from an abstract algebra point of view, it is of interest to observe that $\widehat{B}_{1}$ is a 3 -element algebra: Shafaat [15] showed that, for any 2-element algebra $A, Q(A)$ is a 2-element chain (for related results see [3]).

The above theorem will be obtained as a consequence of a result of [2] stating that if a quasivariety $\mathbf{K}$ of algebras of finite type contains an infinite family of finite algebras indexed by the set of all finite subsets of $\omega$ 
and satisfying certain postulates denoted (P1)-(P4), then the ideal lattice of a free lattice with $\omega$ free generators is embeddable in the lattice of subquasivarieties of $Q(\mathbf{K})$. Not only is Theorem 1.1 a consequence, but it also follows that $Q\left(\widehat{B}_{1}\right)$ is $Q$-universal. A quasivariety $\mathbf{K}$ of algebras of finite type is $Q$-universal if, for every quasivariety $\mathbf{L}$ of algebras of finite type, the lattice of subquasivarieties of $\mathbf{L}$ is a homomorphic image of a sublattice of the lattice of subquasivarieties of $\mathbf{K}$. This notion was introduced by Sapir [12] where the first examples of $Q$-universal quasivarieties were given.

The postulates $(\mathrm{P} 1)-(\mathrm{P} 4)$ will be stated in $\S 2$ and appropriate members of $Q\left(\widehat{B}_{1}\right)$ will be constructed in $\S 3$. Basic properties of these algebras will be established in $\S 4$, enabling the proof in $\S 5$ that they satisfy $(\mathrm{P} 1)-(\mathrm{P} 4)$.

2. Preliminaries. Let $P_{\text {fin }}(\omega)$ denote the set of all finite subsets of $\omega$. We shall need the following.

Proposition 2.1 ([2]). Let $\mathbf{K}$ be a quasivariety of algebras of finite type that contains an infinite family $\left(S_{W}: W \in P_{\text {fin }}(\omega)\right)$ of finite algebras satisfying the following postulates:

(P1) $\quad S_{\emptyset}$ is a trivial algebra;

(P2) for $X \in P_{\text {fin }}(\omega)$, if $X=Y \cup Z$, then $S_{X} \in Q\left(S_{Y}, S_{Z}\right)$;

(P3) for $X, Y \in P_{\text {fin }}(\omega)$, if $X \neq \emptyset$ and $S_{X} \in Q\left(S_{Y}\right)$, then $X=Y$;

(P4) for $X \in P_{\text {fin }}(\omega)$, if $S_{X}$ is a subalgebra of $B \times C$ for finite $B, C \in$ $Q\left(\left\{S_{W}: W \in P_{\text {fin }}(\omega)\right\}\right)$, then there are $Y, Z \in P_{\text {fin }}(\omega)$ with $S_{Y} \in$ $Q(B), S_{Z} \in Q(C)$, and $X=Y \cup Z$.

Then the ideal lattice of a free lattice with $\omega$ generators is embeddable in the lattice of all subquasivarieties of $\mathbf{K}$. In particular, the lattice of all subquasivarieties of $\mathbf{K}$ has a sublattice freely generated by $\omega$ elements and is of cardinality $2^{\omega}$.

Before constructing a subfamily of $Q\left(\widehat{B}_{1}\right)$ satisfying $(\mathrm{P} 1)-(\mathrm{P} 4)$, we note the following terminology.

Let $S$ be a pseudocomplemented semilattice. The skeleton of $S$ is the set $S^{*}=\left\{s^{*}: s \in S\right\}$. Because $s^{* * *}=s^{*}$ for all $s \in S$, the skeleton is the image of the Glivenko endomorphism $\gamma: S \rightarrow S$ given by $\gamma(s)=s^{* *}$ for all $s \in S$. The kernel of $\gamma$ is called the Glivenko congruence $\Gamma$ of $S$, and hence for $s \in S$ the set $[s] \Gamma=\{t \in S: \gamma(t)=\gamma(s)\}$ is known as the Glivenko class of $s$. When $[s] \Gamma$ is finite it has a least element, which we denote as $\mu s$. Because $S^{*}$ is a Boolean algebra (with complementation given by $*$ and join given by $p \vee q=\left(p^{*} \wedge q^{*}\right)^{*}$ for all $\left.p, q \in S^{*}\right)$, if $\varphi: S \rightarrow T$ is a homomorphism (where $T$ is a pseudocomplemented semilattice), then $\varphi\left\lceil S^{*}: S^{*} \rightarrow T^{*}\right.$ is a Boolean homomorphism.

Finally, we mention some notations concerning a partially ordered set $(S ; \leq)$. For $s \in S$, the set $\{t \in S: t \leq s\}$ is denoted by $(s]$, and analogously 
$\{t \in S: t \geq s\}$ is denoted by $[s)$. For $s, t \in S$, the notation $s>t$ will signify that $s$ covers $t$ in the sense that $s>t$ and there is no $u \in S$ satisfying $s>u>t$.

3. The construction. In this section we will construct an infinite family $\left(S_{W}: W \in P_{\text {fin }}(\omega)\right)$ of finite pseudocomplemented semilattices. We begin by defining a finite pseudocomplemented semilattice $\left(S_{m} ; \leq\right)$ for each $m<\omega$.

For $m<\omega$, distinguish three atoms of $B_{m+4}$, denoted by $a, b$, and $c$, and let $d=a \vee b$ and $e=a \vee c$. (We will refer to the element $a$ immediately and to the element $b$ later in this section. However, we shall not refer to the elements $c, d$, and $e$ until $\S 4$.) Let

$$
\begin{aligned}
S_{m}= & \left(B_{m+4} \times \mathbf{3}\right) \\
& \backslash(\{(0,1),(0,2),(a, 2),(1,0),(1,1)\} \cup\{(p, 1): p \neq a \text { and } p \nsucc a\})
\end{aligned}
$$

where 3 denotes the 3 -element chain $\{0<1<2\}$. It is to be shown that $\left(S_{m} ; \leq\right)$, where $\leq$ denotes the restriction of the usual ordering of $B_{m+4} \times \mathbf{3}$ to $S_{m}$, is a pseudocomplemented semilattice. By way of example, $\left(S_{0} ; \leq\right)$ is

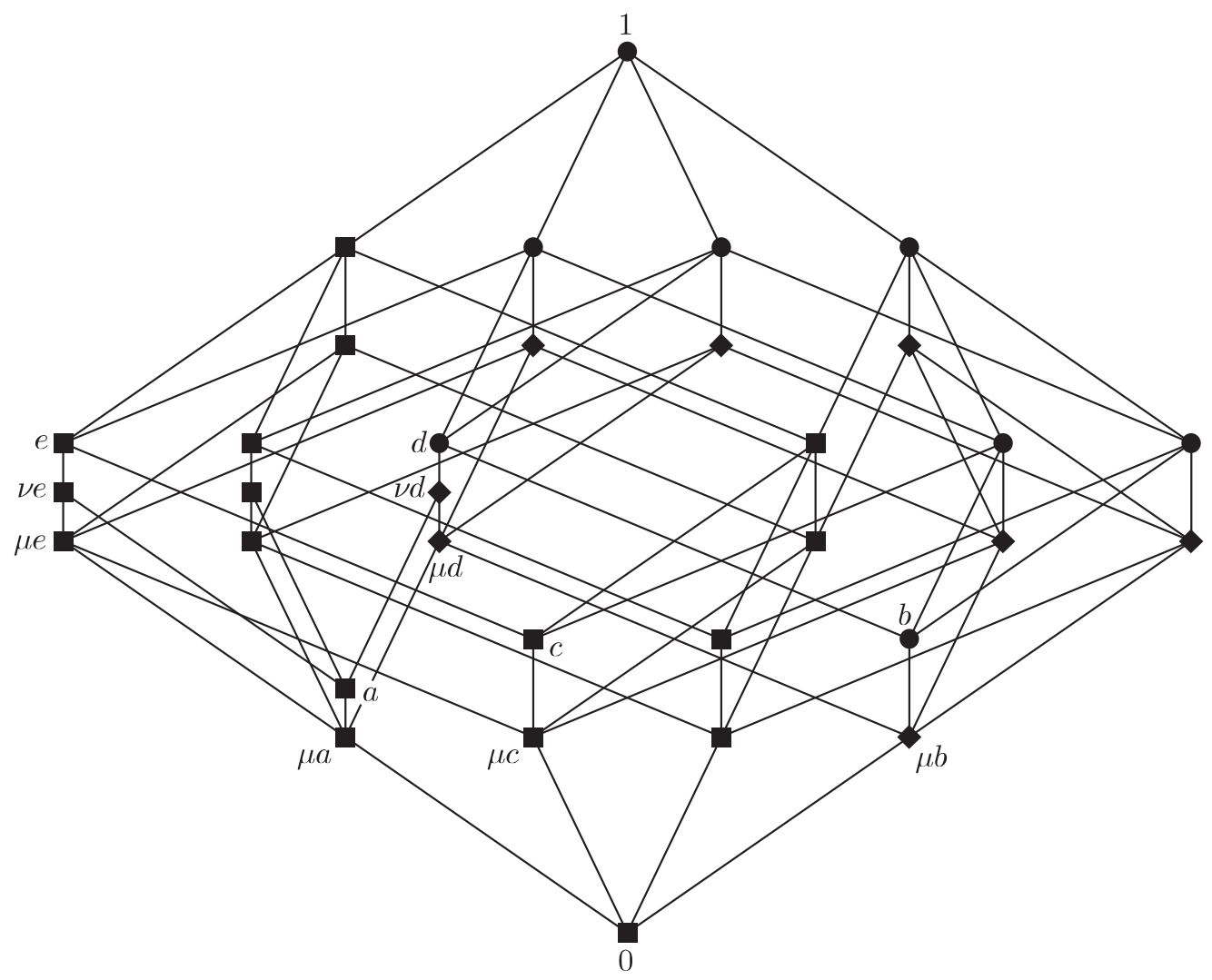

Fig. 1. $S_{0}$ with distinguished elements 
diagrammed in Figure 1; for an explanation of the notation, see the paragraph following Lemma 3.2, and for an explanation of the different shapes used to denote elements, see the first paragraph of $\S 4$.

LEMma 3.1. For $m<\omega,\left(S_{m} ; \leq\right)$ is a semilattice such that, for $(p, i)$, $(q, j) \in S_{m},(p, i) \wedge(q, j)=(p \wedge q, k)$ where $k=0$ if $p \wedge q=0, k=1$ if $p \wedge q=a$ and $i=j=2$, and $i \wedge j$ otherwise.

Proof. For $(p, i),(q, j) \in S_{m}$, consider $(r, k) \in S_{m}$ such that $(r, k) \leq$ $(p, i)$ and $(q, j)$. Clearly, $(r, k) \leq(p \wedge q, i \wedge j)$ and, in the event that $(p \wedge q$, $i \wedge j) \in S_{m},(p, i) \wedge(q, j)=(p \wedge q, i \wedge j)$. Suppose $(p \wedge q, i \wedge j) \notin S_{m}$.

By hypothesis, $p \wedge q \neq 1$.

If $p \wedge q=0$, then $r=0$ and it follows that, as required, $(r, k)=(0,0)$.

If $p \wedge q=a$, then $i \wedge j=2$ and, hence, $i=j=2$. Consequently, $(r, k)=(0,0),(a, 0)$, or $(a, 1)$, whereupon $(p, i) \wedge(q, j)=(a, 1)$.

It remains to consider $p \wedge q \notin\{0, a, 1\}$. To complete the proof it is sufficient to show that (under the hypothesis $\left.(p \wedge q, i \wedge j) \notin S_{m}\right)$ this can never occur. Suppose $p \wedge q \notin\{0, a, 1\}$. Then, since $(p \wedge q, i \wedge j) \notin S_{m}, i \wedge j=1$. Without loss of generality, we may assume $i=1$. Since $(p, i) \in S_{m}$, either $p=a$ or $p>a$. Because $j \geq i$, it would follow that $(p \wedge q, i \wedge j)=(p, i) \in S_{m}$ were $q \geq p$. Thus, $q \geq p$ and, as a consequence, $p \wedge q=a$ or 0 , which is absurd.

Lemma 3.2. For $m<\omega,\left(S_{m} ; \leq\right)$ is a pseudocomplemented semilattice where $(1,2)^{*}=(0,0),\left(a^{*}, 0\right)^{*}=\left(a^{*}, 2\right)^{*}=(a, 1)$, and, for $p \neq a^{*}$ or 1 , $(p, i)^{*}=\left(p^{*}, 2\right)$.

Proof. By Lemma 3.1, for $(p, i),(q, j) \in S_{m},(p, i) \wedge(q, j)=(0,0)$ if and only if $p \wedge q=0$ and, in particular, $q \leq p^{*}$. Consequently, $(p, i)^{*}=\left(p^{*}, 2\right)$, $(0,0)$, or $(a, 1)$ for $p^{*} \neq 0$ or $a, p^{*}=0$, or $p^{*}=a$, respectively.

By Lemma 3.2, for $m<\omega, S_{m}^{*}=\{(0,0),(a, 1)\} \cup\{(p, 2): p \neq 0$ or $a\}$, $|[(0,0)] \Gamma|=|[(1,2)] \Gamma|=1,|[(a, 1)] \Gamma|=2,|[(p, 2)] \Gamma|=3$ if $p>a$, and $|[(p, 2)] \Gamma|=2$ otherwise. Rather than consider $n$-tuples of ordered pairs, we will now, in the forthcoming interests of clarity, identify $(p, i) \in S_{m}^{*}$ with $p$. Thus, $(0,0),(a, 1)$, and $(p, 2)$ will be identified with $0, a$, and $p$, respectively. Further, the smallest element of $[p] \Gamma$ will be denoted by $\mu p$. Thus, $(1,2)$ and $(p, 0)$ for $p \neq 1$ will be identified with $\mu 1$ and $\mu p$, respectively. Finally, if $p \in S_{m}^{*}$ and $|[p] \Gamma|=3$, then the remaining element of $[p] \Gamma$ will be denoted by $\nu p$. To summarize, for $p \in S_{m}^{*}, \mu p \leq p$ where the inequality is strict unless $p=0$ or 1 . Furthermore, $|[p] \Gamma|>2$ if and only if $p>a$, in which case $[p] \Gamma=\{\mu p, \nu p, p\}$ where $\mu p<\nu p<p$.

We shall need the following result in order to show that, for $m<\omega$, $S_{m} \in Q\left(\widehat{B}_{1}\right)$. 
Proposition 3.3 ([13]; cf. [14]). For a finite pseudocomplemented semilattice $(S ; \wedge, *, 0,1), s \in S$, and $\mu s$ the smallest element of $[s] \Gamma$, if $\theta \subseteq S \times S$ is given by $(t, r) \in \theta$ if and only if both $t$ and $r$ belong to $[s),[\mu s) \backslash[s)$, or $S \backslash[\mu s)$ and, in addition, $t^{* *} \wedge s=r^{* *} \wedge s$ also holds for the case $t, r \in S \backslash[\mu s)$, then $\theta$ is a congruence. Furthermore, $S / \theta \cong \widehat{B}_{m}$ for some $m \geq 1$ if and only if $s \neq \mu s$.

For $m<\omega$ and an atom $p$ of $S_{m}^{*}$, let $\theta_{p}$ be the congruence given by Proposition 3.3 with $s=p$. Since $p \neq \mu p$ and $t^{* *} \wedge p=0$ for all $t \in S \backslash[\mu p)$, it follows that if $p$ is an atom of $S_{m}^{*}$, then, for all $t, r \in S_{m},(t, r) \in \theta_{p}$ if and only if both $t$ and $r$ belong to $[p)$, $[\mu p) \backslash[p)$, or to $S_{m} \backslash[\mu p)$. In other words, the equivalence classes of $\theta_{p}$ are $[p),[\mu p) \backslash[p)$, and $S_{m} \backslash[\mu p)$ and, in particular, $S_{m} / \theta_{p} \cong \widehat{B}_{1}$.

Lemma 3.4. For $m<\omega, S_{m} \in Q\left(\widehat{B}_{1}\right)$.

Proof. For $q \in S_{m}^{*}$ distinct from 0 and 1 , there exists an atom $p$ of $S_{m}^{*}$ such that $p \leq q$. In particular, $\mu q \not \equiv q\left(\theta_{p}\right)$. Furthermore, if $q>a$, then $p$ may be chosen so that $q=a \vee p$. It follows that $\nu q \not \equiv q\left(\theta_{p}\right)$ and $\mu q \not \equiv \nu q$ $\left(\theta_{a}\right)$. Since $S_{m}^{*}$ is a Boolean algebra and $S_{m}^{*} \cong S_{m} / \Gamma$, it follows that $S_{m}$ is a subdirect product of suitably many copies of $\widehat{B}_{0}$ and $\widehat{B}_{1}$.

We are now ready to define an infinite family $\left(S_{W}: W \in P_{\text {fin }}(\omega)\right)$ of finite pseudocomplemented semilattices. Recall that, for $m<\omega, b$ is a distinguished atom of $S_{m}^{*}$ distinct from $a$. For $W \in P_{\text {fin }}(\omega)$ where $W=$ $\left\{m_{1}, \ldots, m_{n}\right\}$, let $S_{W}$ denote the subset of $\prod\left(S_{m_{i}}: m_{i} \in W\right)$ whose elements are precisely those $\left(s_{1}, \ldots, s_{n}\right)$ for which $s_{i} \in[b)$ for all $1 \leq i \leq n$, or $s_{i} \in[\mu b) \backslash[b)$ for all $1 \leq i \leq n$, or $s_{i} \in S_{m} \backslash[\mu b)$ for all $1 \leq i \leq n$. It is readily seen that $S_{W}$ is a subalgebra of $\prod\left(S_{m_{i}}: m_{i} \in W\right)$. By Lemma 3.4, for every $W \in P_{\text {fin }}(\omega), S_{W} \in Q\left(\widehat{B}_{1}\right)$.

4. Homomorphisms of $S_{X}$. Let $X$ be a non-empty member of $P_{\text {fin }}(\omega)$. For each $i \in X$ the projection map $\pi_{i}: S_{X} \rightarrow S_{i}$ sends each member of $S_{X}$ to its $i$ th component. Recalling the elements $a, b, c, d, e, 0$ and 1 of each $S_{i}$ we specify elements $\mathbf{a}, \mathbf{b}, \mathbf{c}, \mathbf{d}, \mathbf{e}, \mathbf{0}$, and $\mathbf{1}$ of $S_{X}$ by stipulating that for each $s \in\{a, b, c, d, e, 0,1\}$ the corresponding $\mathbf{s} \in S_{X}$ satisfies $\pi_{i}(\mathbf{s})=s$ for all $i \in X$. Since $S_{X}$ is the subalgebra of $\prod\left(S_{i}: i \in X\right)$ whose elements are those $\mathbf{r} \in \prod\left(S_{i}: i \in X\right)$ for which $\pi_{i}(\mathbf{r}) \in[b)$ for all $i \in X$, or $\pi_{i}(\mathbf{r}) \in[\mu b) \backslash[b)$ for all $i \in X$, or $\pi_{i}(\mathbf{r}) \in S_{i} \backslash[\mu b)$ for all $i \in X$, it is evident that, for $s \in\{a, b, c, d, e, 0,1\}, \mathbf{s} \in S_{X}$. Clearly, for $\mathbf{r} \in S_{X}$, the conditions $\pi_{i}(\mathbf{r}) \in[b)$ for all $i \in X$, or $\pi_{i}(\mathbf{r}) \in[\mu b) \backslash[b)$ for all $i \in X$, or $\pi_{i}(\mathbf{r}) \in S_{i} \backslash[\mu b)$ for all $i \in X$ determine a partition of $S_{X}$. The classes of this partition will be referred to as the $\mathbf{b}$-class, $\mu \mathbf{b}$-class, and $\mathbf{0}$-class, respectively. Evidently, $\mathbf{b}$ and $\mathbf{0}$ are the smallest members of their respective classes. Since $S_{X}$ is 
finite, for every $\mathbf{r} \in S_{X}$, the element $\mu \mathbf{r}$ exists in $S_{X}$. One may also observe that, for any $\mathbf{r} \in S_{X}$, the element $\mu \mathbf{r}$ satisfies $\pi_{i}(\mu \mathbf{r})=\mu \pi_{i}(\mathbf{r})$ for all $i \in X$ unless $\pi_{i}(\mathbf{r})=1$ for some $i \in X$, in which case $\mu \mathbf{r}=\mathbf{r}$. In particular, $\mu \mathbf{b}$ is also the smallest member of its class. Note further that $\nu \mathbf{d} \in S_{X}$ exists where $\pi_{i}(\nu \mathbf{d})=\nu d$ for all $i \in X$. (By way of example, observe that, of the remaining elements distinguished thus far, $\mathbf{d}$ and $\mathbf{1}$ belong to the $\mathbf{b}$-class, $\nu \mathbf{d}$ belongs to the $\mu \mathbf{b}$-class, and $\mathbf{a}, \mathbf{c}$, and e belong to the $\mathbf{0}$-class.) In $S_{m}$, the $b$-class, $\mu$-class, and 0-class are defined likewise. See Figure 1, where, for $S_{0}$, members of its $b$-class, $\mu b$-class, and 0 -class are indicated by circles, diamonds, and boxes, respectively.

Further, for $\mathbf{r} \in S_{X}^{*}$ and $j \in X$ the element $\mu_{j} \mathbf{r} \in \prod\left(S_{i}: i \in X\right)$ is defined so that $\pi_{j}\left(\mu_{j} \mathbf{r}\right)=\mu \pi_{j}(\mathbf{r})$ and $\pi_{i}\left(\mu_{j} \mathbf{r}\right)=\pi_{i}(\mathbf{r})$ for $i \in X \backslash\{j\}$ : since there exist $\mathbf{r} \in S_{X}^{*}$ such that $\mu_{j}(\mathbf{r}) \notin S_{X}$ (for example, $\mathbf{r}=\mathbf{b}$ ), this notation will be used only when $\mu_{j} \mathbf{r} \in S_{X}$. For any Boolean algebra $B$, let $\operatorname{At}(B)$ denote the set of atoms of $B$. Then, for any $j \in X$ and $p \in \operatorname{At}\left(S_{j}^{*}\right) \backslash\{b\}$, $\zeta_{j} \mathbf{p}$ denotes the element of $S_{X}^{*}$ for which $\pi_{j}\left(\zeta_{j} \mathbf{p}\right)=p$ and $\pi_{i}\left(\zeta_{j} \mathbf{p}\right)=0$ for $i \in X \backslash\{j\}$. Note that, for every $j \in X$ and $p \in \operatorname{At}\left(S_{j}^{*}\right) \backslash\{b\}, \zeta_{j} \mathbf{p}$ exists in $S_{X}$ and that the atoms of $S_{X}^{*}$ are precisely all elements of this form together with $\mathbf{b}$.

Finally, for each $i \in \omega$, set $C_{i}(a)=\left\{a \vee p: p \in \operatorname{At}\left(S_{i}^{*}\right) \backslash\{a\}\right\}$. If $\mathbf{r} \in S_{X}^{*}$ satisfies $\pi_{j}(\mathbf{r}) \in C_{j}(a) \backslash\{d\}$ for some $j \in X$, let $\nu_{j} \mathbf{r}$ denote the element of $S_{X}$ defined by $\pi_{j}\left(\nu_{j} \mathbf{r}\right)=\nu \pi_{j}(\mathbf{r})$ and $\pi_{i}\left(\nu_{j} \mathbf{r}\right)=\pi_{i}(\mathbf{r})$ for $i \in X \backslash\{j\}$; since $\pi_{j}(\mathbf{r}) \in C_{j}(a) \backslash\{d\}$ for some $j \in X$, it is always the case that $\nu_{j} \mathbf{r}$ exists in $S_{X}$.

In this section we shall develop properties of homomorphisms defined on $S_{X}$ that separate $\mathbf{e}$ and $\nu_{j} \mathbf{e}$. The key properties are expressed in Propositions 4.3, 4.5, and 4.6.

The first lemma is a clear consequence of the construction of $S_{m}$.

Lemma 4.1. For $m \in \omega, r \in S_{m}^{*}$, and $s \in S_{m} \backslash S_{m}^{*}$, the following hold:

(i) if $r \leq s$, then $r \in\{0, a\}$;

(ii) if $s \geq a$ and $s \in[r] \Gamma$, then $r \in C_{m}(a)$.

Lemma 4.2. Let $X \in P_{\text {fin }}(\omega), j \in X$, and $m \in \omega$. If $\varphi: S_{X} \rightarrow S_{m}$ is a homomorphism such that $\varphi(\mathbf{e}) \neq \varphi\left(\nu_{j} \mathbf{e}\right)$, then the following hold:

(i) $\varphi(\mathbf{a})=a, \varphi(\mathbf{e}) \in C_{m}(a)$, and $\varphi(\mathbf{c}) \in \operatorname{At}\left(S_{m}^{*}\right)$;

(ii) if $\mathbf{r} \in S_{X}^{*}$ satisfies $\pi_{i}(\mathbf{r}) \in C_{i}(a) \backslash\{d\}$ for all $i \in X$, then $\varphi(\mathbf{r}) \neq$ $\varphi\left(\nu_{j} \mathbf{r}\right)$ and $\varphi(\mathbf{r}) \in C_{m}(a)$;

(iii) $\varphi(\mathbf{b}) \neq \varphi(\mu \mathbf{b}), \varphi(\mathbf{d}) \neq \varphi(\nu \mathbf{d}), \varphi(\mathbf{b}) \in \operatorname{At}\left(S_{m}^{*}\right)$, and $\varphi(\mathbf{d}) \in C_{m}(a)$;

(iv) $\varphi\left(\mu \zeta_{j} \mathbf{p}\right) \neq \varphi\left(\zeta_{j} \mathbf{p}\right) \in \operatorname{At}\left(S_{m}^{*}\right)$ for $p \in \operatorname{At}\left(S_{j}^{*}\right) \backslash\{b\}, \varphi\left(\zeta_{i} \mathbf{p}\right)=0$ for $i \in X \backslash\{j\}$ and $p \in \operatorname{At}\left(S_{i}^{*}\right) \backslash\{b\}$, and, in particular, $\varphi\left(\zeta_{j} \mathbf{a}\right)=a$. 
Proof. (i) Because $\varphi(\mathbf{e})$ and $\varphi\left(\nu_{j} \mathbf{e}\right)$ are distinct and share a Glivenko class, $\varphi\left(\nu_{j} \mathbf{e}\right) \in S_{m} \backslash S_{m}^{*}$. Thus, since $\mathbf{a} \leq \nu_{j} \mathbf{e}$, Lemma 4.1(i) implies that $\varphi(\mathbf{a}) \in\{0, a\}$. Suppose $\varphi(\mathbf{a})=0$. Immediately $\varphi\left(\mathbf{a}^{*}\right)=1$, whence the triviality of $[1] \Gamma$ yields $\varphi\left(\mu \mathbf{a}^{*}\right)=1$, and therefore $\varphi(\mu \mathbf{c})=\varphi\left(\mu \mathbf{a}^{*} \wedge \mathbf{c}\right)=$ $\varphi\left(\mu \mathbf{a}^{*}\right) \wedge \varphi(\mathbf{c})=\varphi(\mathbf{c})$, forcing $\varphi\left(\mu_{j} \mathbf{c}\right)=\varphi(\mathbf{c})$. Since $\mu_{j} \mathbf{c}<\nu_{j} \mathbf{e}$, it then follows that $\varphi(\mathbf{c})=\varphi\left(\mu_{j} \mathbf{c}\right) \leq \varphi\left(\nu_{j} \mathbf{e}\right)$, whence $\varphi(\mathbf{c}) \in\{0, a\}$ by Lemma 4.1(i). Inasmuch as the hypothesis entails $0<\varphi(\mathbf{e})=\varphi(\mathbf{a}) \vee \varphi(\mathbf{c})=\varphi(\mathbf{c})$, it follows that $\varphi(\mathbf{c})=a=\varphi(\mathbf{e})$. Thus $a=\varphi(\mathbf{c})=\varphi\left(\mu_{j} \mathbf{c}\right) \leq \varphi\left(\nu_{j} \mathbf{e}\right) \leq$ $\varphi(\mathbf{e})=a$, forcing $\varphi\left(\nu_{j} \mathbf{e}\right)=\varphi(\mathbf{e})$, contrary to hypothesis. Hence, $\varphi(\mathbf{a})=$ $a$. Because $\varphi(\mathbf{e})$ and $\varphi\left(\nu_{j} \mathbf{e}\right)$ are distinct and share a Glivenko class, and $\varphi\left(\nu_{j} \mathbf{e}\right) \geq \varphi(\mathbf{a})=a$, Lemma 4.1(ii) now implies that $\varphi(\mathbf{e}) \in C_{m}(a)$. Since $\varphi(\mathbf{e})=\varphi(\mathbf{a}) \vee \varphi(\mathbf{c})=a \vee \varphi(\mathbf{c})$ and $0=\varphi(\mathbf{0})=\varphi(\mathbf{a}) \wedge \varphi(\mathbf{c})=a \wedge \varphi(\mathbf{c})$, it follows that $\varphi(\mathbf{c}) \in \operatorname{At}\left(S_{m}^{*}\right)$.

(ii) For each $i \in X$ choose $q_{i} \in \operatorname{At}\left(S_{i}^{*}\right) \backslash\{a\}$ such that $\pi_{i}(\mathbf{r})=a \vee q_{i}$. Let q denote the element of $S_{X}$ satisfying $\pi_{i}(\mathbf{q})=q_{i}$ for all $i \in X$ : notice that $\mathbf{q}$ exists in $S_{X}$. If $\varphi(\mathbf{r})=\varphi\left(\nu_{j} \mathbf{r}\right)$, then $\varphi(\mathbf{q})=\varphi(\mathbf{r} \wedge \mathbf{q})=\varphi(\mathbf{r}) \wedge \varphi(\mathbf{q})=\varphi\left(\nu_{j} \mathbf{r}\right) \wedge$ $\varphi(\mathbf{q})=\varphi\left(\nu_{j} \mathbf{r} \wedge \mathbf{q}\right)=\varphi\left(\mu_{j} \mathbf{q}\right)$. Thus, $\varphi\left(\mu_{j} \mathbf{b}^{*}\right) \geq \varphi\left(\mu_{j} \mathbf{q}\right)=\varphi(\mathbf{q})$. Either $\varphi\left(\mu_{j} \mathbf{b}^{*}\right)=\varphi\left(\mathbf{b}^{*}\right)$ or, by Lemma 4.1(i), $\varphi(\mathbf{q}) \in\{0, a\}$. If $\varphi\left(\mu_{j} \mathbf{b}^{*}\right)=\varphi\left(\mathbf{b}^{*}\right)$, then $\varphi(\mathbf{e})=\varphi\left(\mathbf{e} \wedge \mathbf{b}^{*}\right)=\varphi(\mathbf{e}) \wedge \varphi\left(\mathbf{b}^{*}\right)=\varphi(\mathbf{e}) \wedge \varphi\left(\mu_{j} \mathbf{b}^{*}\right)=\varphi\left(\mathbf{e} \wedge \mu_{j} \mathbf{b}^{*}\right)=$ $\varphi\left(\mu_{j} \mathbf{e}\right)$ and, since $\mu_{j} \mathbf{e} \leq \nu_{j} \mathbf{e} \leq \mathbf{e}, \varphi(\mathbf{e})=\varphi\left(\nu_{j} \mathbf{e}\right)$, contrary to hypothesis. If $\varphi(\mathbf{q})=a$, then, by (i), $0=\varphi(\mathbf{0})=\varphi(\mathbf{a} \wedge \mathbf{q})=a \wedge a=a$. Thus, $\varphi(\mathbf{q})=0$. In particular, $\varphi\left(\mathbf{q}^{*}\right)=1$, whence the triviality of $[1] \Gamma$ yields $\varphi\left(\mu \mathbf{q}^{*}\right)=1$, and therefore $\varphi\left(\mathbf{b}^{*}\right)=\varphi\left(\mu \mathbf{q}^{*}\right) \wedge \varphi\left(\mathbf{b}^{*}\right)=\varphi\left(\mu \mathbf{q}^{*} \wedge \mathbf{b}^{*}\right)=\varphi\left(\mu \mathbf{q}^{*} \wedge \mu \mathbf{b}^{*}\right)=$ $\varphi\left(\mu \mathbf{q}^{*}\right) \wedge \varphi\left(\mu \mathbf{b}^{*}\right)=\varphi\left(\mu \mathbf{b}^{*}\right)$. Since $\varphi\left(\mu \mathbf{b}^{*}\right) \leq \varphi\left(\mu_{j} \mathbf{b}^{*}\right) \leq \varphi\left(\mathbf{b}^{*}\right), \varphi\left(\mu_{j} \mathbf{b}^{*}\right)=$ $\varphi\left(\mathbf{b}^{*}\right)$, which we have already seen to be impossible. Thus, $\varphi(\mathbf{r}) \neq \varphi\left(\nu_{j} \mathbf{r}\right)$, whereupon, as $\varphi\left(\nu_{j} \mathbf{r}\right) \geq \varphi(\mathbf{a})=a$, Lemma 4.1(ii) implies that $\varphi(\mathbf{r}) \in C_{m}(a)$.

(iii) For each $i \in X$ choose $q_{i} \in \operatorname{At}\left(S_{i}^{*}\right) \backslash\{a, b, c\}$. Let $\mathbf{q}$ denote the element of $S_{X}$ satisfying $\pi_{i}(\mathbf{q})=q_{i}$ for all $i \in X$. If $\varphi\left(\mathbf{q}^{*}\right)=\varphi\left(\mu \mathbf{q}^{*}\right)$, then, as $\mathbf{q}^{*} \geq \mathbf{e}$, $\varphi(\mathbf{e})=\varphi\left(\mathbf{q}^{*} \wedge \mathbf{e}\right)=\varphi\left(\mathbf{q}^{*}\right) \wedge \varphi(\mathbf{e})=\varphi\left(\mu \mathbf{q}^{*}\right) \wedge \varphi(\mathbf{e})=\varphi\left(\mu \mathbf{q}^{*} \wedge \mathbf{e}\right)=\varphi(\mu \mathbf{e})$, contradicting the hypothesis. Hence, $\varphi\left(\mathbf{q}^{*}\right) \neq \varphi\left(\mu \mathbf{q}^{*}\right)$. If $\varphi(\mathbf{b})=\varphi(\mu \mathbf{b})$, then, as $\varphi\left(\mathbf{q}^{*}\right)>\varphi\left(\mu \mathbf{q}^{*}\right) \geq \varphi(\mu \mathbf{b})=\varphi(\mathbf{b})$, Lemma 4.1(i) implies that $\varphi(\mathbf{b}) \in\{0, a\}$. Since $\varphi(\mathbf{a})=a$ and $\mathbf{a} \wedge \mathbf{b}=\mathbf{0}$, it follows that $\varphi(\mathbf{b})=0$ and, hence, $\varphi\left(\mathbf{b}^{*}\right)=1$. By the triviality of $[1] \Gamma, \varphi\left(\mu \mathbf{b}^{*}\right)=\varphi\left(\mathbf{b}^{*}\right)$ and, arguing as above, $\varphi(\mathbf{e})=\varphi(\mu \mathbf{e})$, in contradiction to the hypothesis. Thus, $\varphi(\mathbf{b}) \neq \varphi(\mu \mathbf{b})$.

If $\varphi(\mathbf{d})=\varphi(\nu \mathbf{d})$, then $\varphi(\mathbf{b})=\varphi(\mathbf{b}) \wedge \varphi(\mathbf{d})=\varphi(\mathbf{b}) \wedge \varphi(\nu \mathbf{d})=\varphi(\mathbf{b} \wedge \nu \mathbf{d})=$ $\varphi(\mu \mathbf{b})$, contradicting the above conclusion. Consequently, $\varphi(\mathbf{d}) \neq \varphi(\nu \mathbf{d})$. Since $\varphi(\nu \mathbf{d}) \in S_{m} \backslash S_{m}^{*}$ and $\varphi(\nu \mathbf{d}) \geq \varphi(\mathbf{a})=a$, Lemma 4.1(ii) implies that $\varphi(\mathbf{d}) \in C_{m}(a)$. Since $\varphi(\mathbf{a} \vee \mathbf{b})=\varphi(\mathbf{d}), \varphi(\mathbf{a} \wedge \mathbf{b})=0, \varphi(\mathbf{a})=a$, and $\varphi(\mathbf{d}) \in C_{m}(a)$, it follows that $\varphi(\mathbf{b}) \in \operatorname{At}\left(S_{m}^{*}\right)$.

(iv) Initially, suppose $p \in \operatorname{At}\left(S_{j}^{*}\right) \backslash\{a, b\}$ and, for $i \in X \backslash\{j\}$, choose $q_{i} \in \operatorname{At}\left(S_{i}^{*}\right) \backslash\{a, b\}$ and let $\mathbf{q}$ denote the element of $S_{X}$ satisfying $\pi_{j}(\mathbf{q})=0$ 
and $\pi_{i}(\mathbf{q})=q_{i}$ for all $i \in X \backslash\{j\}$. By (ii), $\varphi\left(\mathbf{a} \vee\left(\zeta_{j} \mathbf{p} \vee \mathbf{q}\right)\right) \in C_{m}(a)$. Since $\varphi(\mathbf{a})=a$ by (i) and $\varphi\left(\mathbf{a} \wedge\left(\zeta_{j} \mathbf{p} \vee \mathbf{q}\right)\right)=0$, it must be the case that $\varphi\left(\zeta_{j} \mathbf{p} \vee \mathbf{q}\right) \in \operatorname{At}\left(S_{m}^{*}\right) \backslash\{a\}$. Because $\varphi\left(\zeta_{j} \mathbf{p} \wedge \mathbf{q}\right)=0$, it follows that either $\varphi\left(\zeta_{j} \mathbf{p}\right)=0$ and $\varphi(\mathbf{q}) \in \operatorname{At}\left(S_{m}^{*}\right) \backslash\{a\}$ or $\varphi\left(\zeta_{j} \mathbf{p}\right) \in \operatorname{At}\left(S_{m}^{*}\right) \backslash\{a\}$ and $\varphi(\mathbf{q})=0$. Since $\nu_{j}\left(\mathbf{a} \vee\left(\zeta_{j} \mathbf{p} \vee \mathbf{q}\right)\right) \geq \mathbf{q}$ and $\mathbf{q}=\bigvee\left\{\zeta_{i} \mathbf{q}_{i}: i \in X \backslash\{j\}\right\} \in S_{X}^{*}$, (ii) and Lemma 4.1(i) imply $\varphi(\mathbf{q}) \in\{0, a\}$, which, in turn, since $\varphi(\mathbf{a})=a$ (by (i)) and $\mathbf{a} \wedge \mathbf{q}=\mathbf{0}$, yields $\varphi(\mathbf{q})=0$. Hence, for $p \in \operatorname{At}\left(S_{j}^{*}\right) \backslash\{a, b\}$, $\varphi\left(\zeta_{j} \mathbf{p}\right) \in \operatorname{At}\left(S_{m}^{*}\right) \backslash\{a\}$.

Let $\mathbf{q} \in S_{X}$ be given by $\pi_{j}(\mathbf{q})=0$ and $\pi_{i}(\mathbf{q})=a$ for all $i \in X \backslash\{j\}$. Since $\varphi(\mathbf{a})=a, \zeta_{j} \mathbf{a} \vee \mathbf{q}=\mathbf{a}$, and $\zeta_{j} \mathbf{a} \wedge \mathbf{q}=\mathbf{0}$, we have either $\varphi\left(\zeta_{j} \mathbf{a}\right)=a$ and $\varphi(\mathbf{q})=0$ or $\varphi\left(\zeta_{j} \mathbf{a}\right)=0$ and $\varphi(\mathbf{q})=a$. We wish to show that $\varphi(\mathbf{q}) \neq a$. Suppose not and that, to the contrary, $\varphi(\mathbf{q})=a$. As argued in the proof of (ii), $\varphi\left(\mu_{j} \mathbf{b}^{*}\right) \neq \varphi\left(\mathbf{b}^{*}\right)$. However, $\mu_{j} \mathbf{b}^{*} \geq \mathbf{q}$ and, hence, $\varphi\left(\mu_{j} \mathbf{b}^{*}\right) \geq \varphi(\mathbf{q})=a$. By Lemma 4.1(ii), $\varphi\left(\mathbf{b}^{*}\right) \in C_{m}(a)$. Since $\mathbf{b} \vee \mathbf{b}^{*}=\mathbf{1}$ and $\varphi(\mathbf{b}) \in \operatorname{At}\left(S_{m}^{*}\right)$ (see (iii)), it follows that the unit in $S_{m}^{*}$ is a join of 3 atoms, which is impossible by the definition of $S_{m}$. Hence, $\varphi(\mathbf{q}) \neq a$ and, as required, $\varphi\left(\zeta_{j} \mathbf{a}\right)=a$. Thus, for $p \in \operatorname{At}\left(S_{j}^{*}\right) \backslash\{b\}, \varphi\left(\zeta_{j} \mathbf{p}\right) \in \operatorname{At}\left(S_{m}^{*}\right)$.

Let $i \in X \backslash\{j\}$ and $p \in \operatorname{At}\left(S_{i}^{*}\right) \backslash\{b\}$. If $p \neq a$, then choose $p^{\prime} \in$ $\operatorname{At}\left(S_{j}^{*}\right) \backslash\{a, b\}$ and $q_{k} \in \operatorname{At}\left(S_{k}^{*}\right) \backslash\{a, b\}$ for $k \in X \backslash\{i, j\}$. Let $\mathbf{q}$ be given by $\pi_{i}(\mathbf{q})=p, \pi_{j}(\mathbf{q})=0$, and $\pi_{k}(\mathbf{q})=q_{k}$ for $k \in X \backslash\{i, j\}$. Then, arguing as above, $\varphi\left(\zeta_{j} \mathbf{p}^{\prime} \vee \mathbf{q}\right) \in \operatorname{At}\left(S_{m}^{*}\right)$, which in turn (see the above) implies $\varphi(\mathbf{q})=0$. Hence, $\zeta_{i} \mathbf{p} \leq \mathbf{q}$ implies $\varphi\left(\zeta_{i} \mathbf{p}\right)=0$. Were $p=a$, let $\pi_{j}(\mathbf{q})=0$ and $\pi_{i}(\mathbf{q})=a$ for all $i \in X \backslash\{j\}$. Since $\zeta_{j} \mathbf{a} \vee \mathbf{q}=\mathbf{a}, \zeta_{j} \mathbf{a} \wedge \mathbf{q}=\mathbf{0}$, and $\varphi(\mathbf{a})=\varphi\left(\zeta_{j} \mathbf{a}\right)=a$, we have $\varphi(\mathbf{q})=0$. Thus, $\zeta_{i} \mathbf{a} \leq \mathbf{q}$ yields $\varphi\left(\zeta_{i} \mathbf{a}\right)=0$. Hence, for $i \in X \backslash\{j\}$ and $p \in \operatorname{At}\left(S_{i}^{*}\right) \backslash\{b\}, \varphi\left(\zeta_{i} \mathbf{p}\right)=0$.

As already seen, $\varphi(\mathbf{e}) \neq \varphi\left(\nu_{j} \mathbf{e}\right)$ implies $\varphi\left(\mathbf{b}^{*}\right) \neq \varphi\left(\mu_{j} \mathbf{b}^{*}\right)$. Since $\mu \zeta_{j} \mathbf{p} \leq$ $\mu_{j} \mathbf{b}^{*}$ for $p \in \operatorname{At}\left(S_{j}^{*}\right) \backslash\{b\}$, if $\varphi\left(\mu \zeta_{j} \mathbf{p}\right)=\varphi\left(\zeta_{j} \mathbf{p}\right)$, then $\varphi\left(\mathbf{b}^{*}\right) \in C_{m}(a)$ by Lemma 4.1(i), (ii). As argued above, this is impossible since the unit of $S_{m}^{*}$ is not the join of 3 atoms. Thus, for $p \in \operatorname{At}\left(S_{j}^{*}\right) \backslash\{b\}, \varphi\left(\mu \zeta_{j} \mathbf{p}\right) \neq \varphi\left(\zeta_{j} \mathbf{p}\right)$.

Proposition 4.3. Let $X \in P_{\text {fin }}(\omega), j \in X$, and $m \in \omega$. If $\varphi: S_{X} \rightarrow S_{m}$ is a homomorphism such that $\varphi(\mathbf{e}) \neq \varphi\left(\nu_{j} \mathbf{e}\right)$, then $j=m$ and there is an automorphism $\eta$ of $S_{m}$ such that $\varphi=\eta \pi_{m}$.

Proof. Because $\operatorname{At}\left(S_{X}^{*}\right)=\{\mathbf{b}\} \cup\left\{\zeta_{i} \mathbf{p}: p \in \operatorname{At}\left(S_{i}^{*}\right) \backslash\{b\}\right.$ and $\left.i \in X\right\}$, $1=\varphi(\mathbf{1})=\varphi\left(\bigvee \operatorname{At}\left(S_{X}^{*}\right)\right)=\varphi(\mathbf{b}) \vee \bigvee\left\{\varphi\left(\zeta_{i} \mathbf{p}\right): p \in \operatorname{At}\left(S_{i}^{*}\right) \backslash\{b\}\right.$ and $\left.i \in X\right\}$, which, by Lemma $4.2\left(\right.$ iv), is $\varphi(\mathbf{b}) \vee \bigvee\left\{\varphi\left(\zeta_{j} \mathbf{p}\right): p \in \operatorname{At}\left(S_{j}^{*}\right) \backslash\{b\}\right\}$. Also by Lemma 4.2(iii), (iv), each of $\varphi(\mathbf{b})$ and $\varphi\left(\zeta_{j} \mathbf{p}\right)$ for $p \in \operatorname{At}\left(S_{j}^{*}\right) \backslash\{b\}$ is a distinct element of $\operatorname{At}\left(S_{m}^{*}\right)$. Thus, the unit element of $S_{m}^{*}$ is a join of $\left|\operatorname{At}\left(S_{j}^{*}\right)\right|$ atoms, whence $S_{j}^{*}$ and $S_{m}^{*}$ have the same number of atoms, whereupon $j=m$.

We now show that, for $\mathbf{r}, \mathbf{s} \in S_{X}, \varphi(\mathbf{r})=\varphi(\mathbf{s})$ if and only if $\pi_{j} \mathbf{r}=\pi_{j} \mathbf{s}$.

If $\mathbf{r} \in S_{X}^{*}$, then $\mathbf{r}$ is the join of all members of $\operatorname{At}\left(S_{X}^{*}\right)$ that lie below it. Let $I=\left\{p \in \operatorname{At}\left(S_{j}^{*}\right) \backslash\{b\}: \zeta_{j} \mathbf{p} \leq \mathbf{r}\right\}$. Then, as above, it follows from Lemma 
4.2(iv) that $\varphi(\mathbf{r})=\varphi(\mathbf{b}) \bigvee \bigvee\left\{\varphi\left(\zeta_{j} \mathbf{p}\right): p \in I\right\}$ or $\bigvee\left\{\varphi\left(\zeta_{j} \mathbf{p}\right): p \in I\right\}$ depending on whether $\mathbf{r}$ is a member of the $\mathbf{b}$-class or not. Similarly, for $\mathbf{s} \in S_{X}^{*}$, if $J=\left\{p \in \operatorname{At}\left(S_{j}^{*}\right) \backslash\{b\}: \zeta_{j} \mathbf{p} \leq \mathbf{s}\right\}$, then $\varphi(\mathbf{s})=\varphi(\mathbf{b}) \vee \bigvee\left\{\varphi\left(\zeta_{j} \mathbf{p}\right): p \in J\right\}$ or $\bigvee\left\{\varphi\left(\zeta_{j} \mathbf{p}\right): p \in J\right\}$ depending on whether $\mathbf{s}$ is a member of the $\mathbf{b}$-class or not. In particular, it follows from Lemma 4.2(iii), (iv) that $\varphi(\mathbf{r})=\varphi(\mathbf{s})$ if and only if $I=J$ and $\mathbf{r} \geq \mathbf{b}$ is equivalent to $\mathbf{s} \geq \mathbf{b}$. Thus, for $\mathbf{r}, \mathbf{s} \in S_{X}^{*}$, $\varphi(\mathbf{r})=\varphi(\mathbf{s})$ if and only if $\pi_{j} \mathbf{r}=\pi_{j} \mathbf{s}$.

Let $\mathbf{r}, \mathbf{s} \in S_{X}$. If $\pi_{j}\left(\mathbf{r}^{* *}\right) \neq \pi_{j}\left(\mathbf{s}^{* *}\right)$, then, by the above, $\varphi\left(\mathbf{r}^{* *}\right) \neq \varphi\left(\mathbf{s}^{* *}\right)$, which implies that $\varphi(\mathbf{r}) \neq \varphi(\mathbf{s})$. Assume $\pi_{j}\left(\mathbf{r}^{* *}\right)=\pi_{j}\left(\mathbf{s}^{* *}\right)$. If $\pi_{j}(\mathbf{r}) \neq \pi_{j}(\mathbf{s})$ and, say, $\pi_{j}(\mathbf{r})>\pi_{j}(\mathbf{s})$, then either $\mathbf{r} \geq \mathbf{b}$ and $\mathbf{s} \wedge \mathbf{b}=\mu \mathbf{b}$ or there exists $p \in \operatorname{At}\left(S_{j}^{*}\right) \backslash\{b\}$ such that $\mathbf{r} \geq \zeta_{j} \mathbf{p}$ and $\mathbf{s} \wedge \zeta_{j} \mathbf{p}=\mu \zeta_{j} \mathbf{p}$. By Lemma 4.2(iii) or (iv), respectively, $\varphi(\mathbf{r}) \neq \varphi(\mathbf{s})$. It remains to show that $\varphi(\mathbf{r})=\varphi(\mathbf{s})$ whenever $\pi_{j}(\mathbf{r})=\pi_{j}(\mathbf{s})$. Suppose $\pi_{j}(\mathbf{r})=\pi_{j}(\mathbf{s})$ and $\varphi(\mathbf{r}) \neq \varphi(\mathbf{s})$. By the above, $\varphi(\mathbf{r}) \equiv \varphi(\mathbf{s})(\Gamma)$. Say, with no loss in generality, $\varphi(\mathbf{r})>\varphi(\mathbf{s})$. Hence, there exists $p \in \operatorname{At}\left(S_{m}^{*}\right)$ such that $\varphi(\mathbf{r}) \geq p$ and $\varphi(\mathbf{s}) \wedge p=\mu p$. Since $S_{X} / \operatorname{Ker}\left(\pi_{j}\right) \cong$ $S_{j}$ where $\operatorname{Ker}\left(\pi_{j}\right)$ denotes the congruence kernel of the homomorphism $\pi_{j}$, $j=m$, and, as seen above, $\operatorname{Ker}(\varphi) \leq \operatorname{Ker}\left(\pi_{j}\right)$ where $\operatorname{Ker}(\varphi)$ denotes the congruence kernel of the homomorphism $\varphi$, it follows that $\varphi$ is onto. Thus, $p=\varphi(\mathbf{t})$ for some $\mathbf{t} \in S_{X}^{*}$. Since $p \in \operatorname{At}\left(S_{m}^{*}\right)$ and $\mathbf{t}$ is the join of all members of $\operatorname{At}\left(S_{X}^{*}\right)$ that lie below it, $p=\varphi(\mathbf{u})$ for some $\mathbf{u} \in \operatorname{At}\left(S_{X}^{*}\right)$. This, by Lemma 4.2(iii), (iv), implies that either $\varphi(\mathbf{b})=p$ or there exists $q \in \operatorname{At}\left(S_{j}^{*}\right)$ such that $\varphi\left(\zeta_{j} \mathbf{q}\right)=p$. Since $\pi_{j}(\mathbf{r})=\pi_{j}(\mathbf{s})$, we have $\mathbf{r} \wedge \mathbf{b}=\mathbf{s} \wedge \mathbf{b}$ or $\mathbf{r} \wedge \zeta_{j} \mathbf{q}=\mathbf{s} \wedge \zeta_{j} \mathbf{q}$, respectively. Thus, $p=\varphi(\mathbf{r} \wedge \mathbf{b})=\varphi(\mathbf{s} \wedge \mathbf{b})=\mu p$ or $p=\varphi\left(\mathbf{r} \wedge \zeta_{j} \mathbf{q}\right)=\varphi\left(\mathbf{s} \wedge \zeta_{j} \mathbf{q}\right)=\mu p$, which is absurd.

Define $\eta: S_{m} \rightarrow S_{m}$ by $\eta(r)=\varphi(\mathbf{s})$, where $\mathbf{s}$ is any element of $S_{X}$ with $\pi_{m}(\mathbf{s})=r$. Since $m=j$ and, for all $\mathbf{r}$ and $\mathbf{s} \in S_{X}, \varphi(\mathbf{r})=\varphi(\mathbf{s})$ if and only if $\pi_{j}(\mathbf{r})=\pi_{j}(\mathbf{s}), \eta$ is well-defined and indeed is an automorphism. Clearly, as required, $\varphi=\eta \pi_{m}$.

In the following lemma the term quasi-atom refers to any $\mathbf{r} \in S_{X}$ such that $\pi_{i}(\mathbf{r}) \in \operatorname{At}\left(S_{i}^{*}\right)$ for all $i \in X$.

Lemma 4.4. Let $X, Y \in P_{\text {fin }}(\omega), j \in X$, and $n \in Y$. If $\psi: S_{X} \rightarrow S_{Y}$ is a homomorphism such that $\psi(\mathbf{e}) \neq \psi\left(\nu_{j} \mathbf{e}\right)$, then the following hold:

(i) if $\mathbf{r}$ is a quasi-atom of $S_{X}$, then $\pi_{n} \psi(\mathbf{r}) \neq 0$ and either $\pi_{n} \psi\left(\mathbf{r}^{*}\right) \neq$ $\pi_{n} \psi\left(\mu \mathbf{r}^{*}\right)$ or $\pi_{n} \psi\left(\mathbf{r}^{*}\right) \leq b^{*}$;

(ii) there exists a quasi-atom $\mathbf{p}$ of $S_{X}$ such that $\mathbf{p} \in\left(\mathbf{b}^{*}\right]$ and $\pi_{n} \psi\left(\mathbf{p}^{*}\right) \neq$ $\pi_{n} \psi\left(\mu \mathbf{p}^{*}\right)$.

Proof. (i) Because $\psi(\mathbf{e}) \neq \psi\left(\nu_{j} \mathbf{e}\right)$, there exists $m \in Y$ such that $\pi_{m} \psi(\mathbf{e}) \neq \pi_{m} \psi\left(\nu_{j} \mathbf{e}\right)$. Proposition 4.3, when applied to the homomorphism $\pi_{m} \psi: S_{X} \rightarrow S_{m}$, implies that $j=m$ and there is an automorphism $\eta$ of $S_{m}$ 
such that $\pi_{m} \psi=\eta \pi_{m}$. (The indicated projection maps have domains $S_{Y}$ and $S_{X}$, respectively.) In particular, $\pi_{m} \psi\left(\mu \mathbf{r}^{*}\right)=\eta \pi_{m}\left(\mu \mathbf{r}^{*}\right) \in S_{m} \backslash S_{m}^{*}$.

If $\pi_{n} \psi(\mathbf{r})=0$, then $\pi_{n} \psi\left(\mathbf{r}^{*}\right)=1$, whence $\pi_{n} \psi\left(\mu \mathbf{r}^{*}\right)=1$ by the triviality of [1] $\Gamma$. Thus, $\psi\left(\mu \mathbf{r}^{*}\right)$ belongs to the $\mathbf{b}$-class of $S_{Y}$, whence $\pi_{m} \psi\left(\mu \mathbf{r}^{*}\right)$ belongs to the $b$-class of $S_{m}$, which is a subset of $S_{m}^{*}$. Since this conclusion contradicts our finding that $\pi_{m} \psi\left(\mu \mathbf{r}^{*}\right) \in S_{m} \backslash S_{m}^{*}$, we have $\pi_{n} \psi(\mathbf{r}) \neq 0$.

If $\pi_{n} \psi\left(\mathbf{r}^{*}\right)=\pi_{n} \psi\left(\mu \mathbf{r}^{*}\right)$, then $\pi_{n} \psi\left(\mu \mathbf{r}^{*}\right) \in S_{n}^{*}$, and since $\pi_{m} \psi\left(\mu \mathbf{r}^{*}\right) \in S_{m} \backslash$ $S_{m}^{*}$, it follows that $\psi\left(\mu \mathbf{r}^{*}\right)$ belongs to the $\mathbf{0}$-class of $S_{Y}$. That is, $\psi\left(\mu \mathbf{r}^{*}\right) \leq \mathbf{b}^{*}$, and therefore $\psi\left(\mathbf{r}^{*}\right) \leq \mathbf{b}^{*}$. Thus $\pi_{n} \psi\left(\mathbf{r}^{*}\right) \neq \pi_{n} \psi\left(\mu \mathbf{r}^{*}\right)$ or $\pi_{n} \psi\left(\mathbf{r}^{*}\right) \leq b^{*}$.

(ii) Because $\operatorname{At}\left(S_{i}^{*}\right) \backslash\{b\} \subseteq\left(b^{*}\right]$ for all $i \in X$, there exist in $S_{X}$ quasiatoms $\mathbf{r}, \mathbf{s} \in\left(\mathbf{b}^{*}\right]$ such that $\mathbf{r} \wedge \mathbf{s}=\mathbf{0}$. Since $1=\pi_{n} \psi(\mathbf{1})=\pi_{n} \psi\left(\mathbf{r}^{*}\right) \vee \pi_{n} \psi\left(\mathbf{s}^{*}\right)$, it is impossible to have both $\pi_{n} \psi\left(\mathbf{r}^{*}\right) \leq b^{*}$ and $\pi_{n} \psi\left(\mathbf{s}^{*}\right) \leq b^{*}$, whereupon (i) yields $\mathbf{p} \in\{\mathbf{r}, \mathbf{s}\}$ such that $\pi_{n} \psi\left(\mathbf{p}^{*}\right) \neq \pi_{n} \psi\left(\mu \mathbf{p}^{*}\right)$.

Proposition 4.5. Let $X, Y \in P_{\text {fin }}(\omega)$ and $j \in X$. If there is a homomorphism $\psi: S_{X} \rightarrow S_{Y}$ such that $\psi(\mathbf{e}) \neq \psi\left(\nu_{j} \mathbf{e}\right)$, then $j \in Y$ and $Y \subseteq X$.

Proof. If $\psi(\mathbf{e}) \neq \psi\left(\nu_{j} \mathbf{e}\right)$, then there exists $m \in Y$ such that $\pi_{m} \psi(\mathbf{e}) \neq$ $\pi_{m} \psi\left(\nu_{j} \mathbf{e}\right)$. Hence, for the homomorphism $\pi_{m} \psi: S_{X} \rightarrow S_{m}$, Proposition 4.3 implies that $j=m$ and, in particular, $j \in Y$. Likewise, to obtain $Y \subseteq X$ by means of Proposition 4.3 it suffices to prove that for every $n \in Y$ there exists $i \in X$ such that $\pi_{n} \psi(\mathbf{e}) \neq \pi_{n} \psi\left(\nu_{i} \mathbf{e}\right)$ and, so, $i=n \in X$.

Suppose, on the contrary, that there exists $n \in Y$ such that $\pi_{n} \psi(\mathbf{e})=$ $\pi_{n} \psi\left(\nu_{i} \mathbf{e}\right)$ for all $i \in X$. For notational convenience set $\varphi=\pi_{n} \psi$.

For $\nu \mathbf{e}=\bigwedge\left\{\nu_{i} \mathbf{e}: i \in X\right\}$, we have $\varphi(\mathbf{e})=\varphi(\nu \mathbf{e})$. It follows that $\varphi(\mathbf{c}) \leq$ $\varphi\left(\mu \mathbf{b}^{*}\right)$ because $\varphi(\mathbf{c})=\varphi(\mathbf{e}) \wedge \varphi(\mathbf{c})=\varphi(\nu \mathbf{e}) \wedge \varphi(\mathbf{c})=\varphi(\mu \mathbf{c}) \leq \varphi\left(\mu \mathbf{b}^{*}\right)$.

Next we show that $\varphi\left(\mathbf{b}^{*}\right)=\varphi\left(\mu \mathbf{b}^{*}\right)$. Supposing the contrary, $\varphi\left(\mu \mathbf{b}^{*}\right) \in$ $S_{n} \backslash S_{n}^{*}$. Since $\varphi(\mathbf{c}) \leq \varphi\left(\mu \mathbf{b}^{*}\right)$ it now follows from Lemma 4.1(i) that $\varphi(\mathbf{c}) \in$ $\{0, a\}$, hence Lemma 4.4(i) yields $\varphi(\mathbf{c})=a$, whereupon, by Lemma 4.1(ii), $\varphi\left(\mathbf{b}^{*}\right) \in C_{n}(a)$ and, hence, there exists $p \in \operatorname{At}\left(S_{n}^{*}\right) \backslash\{a\}$ such that $\varphi\left(\mathbf{b}^{*}\right)=$ $a \vee p$. Since $\mathbf{a} \leq \mathbf{b}^{*}$, it follows that $\varphi(\mathbf{a}) \in\{0, a, p, a \vee p\}$. Inasmuch as $\varphi(\mathbf{a}) \neq 0$ by Lemma 4.4(i), and $0=\varphi(\mathbf{a}) \wedge \varphi(\mathbf{c})=\varphi(\mathbf{a}) \wedge a$, we conclude that $\varphi(\mathbf{a})=p$. Choose a quasi-atom $\mathbf{r}$ of $S_{X}$ such that, for all $i \in X, \pi_{i}(\mathbf{r}) \notin$ $\{a, b, c\}$. It follows that $\mathbf{r} \leq \mathbf{b}^{*}$ and, hence, $\varphi(\mathbf{r}) \leq \varphi\left(\mathbf{b}^{*}\right)$. By Lemma 4.4(i), $\varphi(\mathbf{r}) \in\{a, p, a \vee p\}$. Since $\varphi(\mathbf{a})=p, \varphi(\mathbf{c})=a, \varphi(\mathbf{r}) \wedge \varphi(\mathbf{a})=\varphi(\mathbf{r} \wedge \mathbf{a})=0$, and $\varphi(\mathbf{r}) \wedge \varphi(\mathbf{c})=\varphi(\mathbf{r} \wedge \mathbf{c})=0$, this is absurd. Therefore $\varphi\left(\mathbf{b}^{*}\right)=\varphi\left(\mu \mathbf{b}^{*}\right)$.

For any $\mathbf{s} \in S_{X}$ such that $\mathbf{s} \leq \mathbf{b}^{*}$ we now have $\varphi(\mathbf{s})=\varphi(\mu \mathbf{s})$. (Indeed, $\varphi(\mu \mathbf{s})=\varphi(\mathbf{s}) \wedge \varphi\left(\mu \mathbf{b}^{*}\right)=\varphi(\mathbf{s}) \wedge \varphi\left(\mathbf{b}^{*}\right)=\varphi(\mathbf{s})$.) In particular, $\varphi(\mathbf{s})=\varphi(\mu \mathbf{s})$ for $\mathbf{s}=\mathbf{p}$, where $\mathbf{p}$ is a quasi-atom obtained from Lemma 4.4(ii). This contradiction concludes the proof.

Proposition 4.6. Let $X \in P_{\text {fin }}(\omega)$ and $m \in \omega$. If $\varphi: S_{X} \rightarrow S_{m}$ is a homomorphism such that $\varphi(\mathbf{e})=\varphi\left(\nu_{i} \mathbf{e}\right)$ for all $i \in X$, then $\Gamma_{X} \leq \operatorname{Ker}(\varphi)$ 
where $\Gamma_{X}$ denotes the Glivenko congruence on $S_{X}$ and $\operatorname{Ker}(\varphi)$ denotes the congruence kernel of the homomorphism $\varphi$.

Proof. Suppose $\varphi\left(\mathbf{b}^{*}\right) \neq \varphi\left(\mu \mathbf{b}^{*}\right)$. Since $\mu \mathbf{b}^{*}=\bigwedge\left\{\mu_{i} \mathbf{b}^{*}: i \in X\right\}$, $\varphi\left(\mathbf{b}^{*}\right) \neq \varphi\left(\mu_{i} \mathbf{b}^{*}\right)$ for some $i \in X$. By hypothesis $\varphi(\mathbf{e})=\varphi\left(\nu_{i} \mathbf{e}\right)$ and, consequently, $\nu_{i} \mathbf{e} \wedge \mathbf{c}=\mu_{i} \mathbf{c}$ implies $\varphi(\mathbf{c})=\varphi(\mathbf{e} \wedge \mathbf{c})=\varphi(\mathbf{e}) \wedge \varphi(\mathbf{c})=\varphi\left(\nu_{i} \mathbf{e}\right) \wedge \varphi(\mathbf{c})=$ $\varphi\left(\nu_{i} \mathbf{e} \wedge \mathbf{c}\right)=\varphi\left(\mu_{i} \mathbf{c}\right)$. Since $\mu_{i} \mathbf{c} \leq \mu_{i} \mathbf{b}^{*}, \varphi(\mathbf{c})=\varphi\left(\mu_{i} \mathbf{c}\right) \leq \varphi\left(\mu_{i} \mathbf{b}^{*}\right) \in S_{m} \backslash S_{m}^{*}$. By Lemma 4.1(i), $\varphi(\mathbf{c})=0$ or $\varphi(\mathbf{c})=a$. Further, if $\varphi(\mathbf{c})=a$, then it follows from Lemma 4.1(ii) that $\varphi\left(\mathbf{b}^{*}\right) \in C_{m}(a)$ and, since $\varphi(\mathbf{c}) \leq \varphi\left(\mu_{i} \mathbf{b}^{*}\right)$, $\varphi\left(\mu_{i} \mathbf{b}^{*}\right)=\nu \varphi\left(\mathbf{b}^{*}\right)$.

We show that neither $\varphi(\mathbf{c})=0$ nor $\varphi(\mathbf{c})=a$ may occur.

If $\varphi(\mathbf{c})=0$, then $\varphi\left(\mathbf{c}^{*}\right)=1$. The triviality of $[1] \Gamma$ reveals that $\varphi\left(\mathbf{c}^{*}\right)=$ $\varphi\left(\mu \mathbf{c}^{*}\right)$. For every quasi-atom $\mathbf{r}$ such that $\pi_{j}(\mathbf{r}) \in \operatorname{At}\left(S_{j}^{*}\right) \backslash\{b, c\}$ for all $j \in X, \mathbf{r} \leq \mathbf{c}^{*}$ and $\mathbf{r} \wedge \mu \mathbf{c}^{*}=\mu \mathbf{r}$. Thus, $\varphi(\mathbf{r})=\varphi(\mu \mathbf{r})$ and, in particular, $\varphi\left(\mu_{i} \mathbf{r}\right)=\varphi(\mathbf{r})$. From $\mathbf{b}^{*} \geq \mathbf{r}$ and $\mu_{i} \mathbf{b}^{*} \geq \mu_{i} \mathbf{r}$ it follows, by Lemma 4.1(i), that $\varphi(\mathbf{r})=0$ or $\varphi(\mathbf{r})=a$. Further, in the event that $\varphi(\mathbf{r})=a$, then, by Lemma 4.1(ii), $\varphi\left(\mathbf{b}^{*}\right) \in C_{m}(a)$. Since $\mathbf{b}^{*}=\mathbf{c} \vee \bigvee\left\{\mathbf{r}: \pi_{j}(\mathbf{r}) \in \operatorname{At}\left(S_{j}^{*}\right) \backslash\{b, c\}\right.$ for all $j \in X$ \}, either $\varphi\left(\mathbf{b}^{*}\right)=0$ or $\varphi\left(\mathbf{b}^{*}\right)=a$. Because $\varphi\left(\mathbf{b}^{*}\right) \neq \varphi\left(\mu \mathbf{b}^{*}\right)$, it follows that $\varphi \mathbf{b}^{*} \neq 0$. Thus, $\varphi\left(\mathbf{b}^{*}\right)=a$. However, this is absurd since $\varphi\left(\mathbf{b}^{*}\right)=a$ only if $\varphi(\mathbf{r})=a$ for some suitable $\mathbf{r}$, which, in turn, implies $\varphi\left(\mathbf{b}^{*}\right) \in C_{m}(a)$. Hence, contrary to the supposition, $\varphi(\mathbf{c}) \neq 0$.

If $\varphi(\mathbf{c})=a, \varphi\left(\mathbf{b}^{*}\right) \in C_{m}(a)$, and $\varphi\left(\mu_{i} \mathbf{b}^{*}\right)=\nu \varphi\left(\mathbf{b}^{*}\right)$, then, for some $p \in \operatorname{At}\left(S_{m}^{*}\right), \varphi\left(\mathbf{b}^{*}\right)=a \vee p$ and $\varphi(\mathbf{a}) \in\{0, a, p, a \vee p\}$. Since $\mathbf{a} \wedge \mathbf{c}=\mathbf{0}$, it follows that $\varphi(\mathbf{a}) \in\{0, p\}$. If $\varphi(\mathbf{a})=0$, then $\varphi\left(\mathbf{a}^{*}\right)=1$. Arguing as above, $\varphi\left(\mathbf{a}^{*}\right)=\varphi\left(\mu \mathbf{a}^{*}\right), \varphi(\mathbf{r})=\varphi\left(\mu_{i} \mathbf{r}\right)$ for every quasi-atom $\mathbf{r}$ such that $\pi_{j}(\mathbf{r}) \in \operatorname{At}\left(S_{j}^{*}\right) \backslash\{a, b\}$, and, hence, $\varphi(\mathbf{r})=0$ or $a$. As before, it follows that $\varphi\left(\mathbf{b}^{*}\right)=a$, which is absurd. Thus, $\varphi(\mathbf{a})=p$ and, as $\mathbf{a} \wedge \mu_{i} \mathbf{b}^{*}=\mu_{i} \mathbf{a}$ and $\varphi\left(\mu_{i} \mathbf{b}^{*}\right)=\nu \varphi\left(\mathbf{b}^{*}\right), \varphi\left(\mu_{i} \mathbf{a}\right)=\varphi(\mathbf{a}) \wedge \varphi\left(\mu_{i} \mathbf{b}^{*}\right)=p \wedge \nu \varphi\left(\mathbf{b}^{*}\right)=\mu p$. In particular, $\varphi\left(\mu_{i} \mathbf{a}\right) \neq \varphi(\mathbf{a})$. Choose a quasi-atom $\mathbf{r}$ such that $\pi_{j}(\mathbf{r}) \in$ $\operatorname{At}\left(S_{j}^{*}\right) \backslash\{a, b, c\}$. Since $\mathbf{r} \leq \mathbf{b}^{*}, \varphi(\mathbf{r}) \in\{0, a, p, a \vee p\}$ and, as $\mathbf{a} \wedge \mathbf{r}=\mathbf{c} \wedge \mathbf{r}=\mathbf{0}$, $\varphi(\mathbf{r})=0$. Hence, $\varphi\left(\mathbf{r}^{*}\right)=1, \varphi\left(\mu_{i} \mathbf{r}^{*}\right)=\varphi\left(\mathbf{r}^{*}\right)$ and, since $\mathbf{r}^{*} \geq \mathbf{a}$, it follows that $\varphi\left(\mu_{i} \mathbf{a}\right)=\varphi(\mathbf{a})$, which is absurd. Thus, contrary to hypothesis, $\varphi(\mathbf{c}) \neq a$.

We conclude that $\varphi\left(\mathbf{b}^{*}\right)=\varphi\left(\mu \mathbf{b}^{*}\right)$.

Since $\mathbf{r} \leq \mathbf{b}^{*}$ for every $\mathbf{r}$ in the $\mathbf{0}$-class, it remains to show that, for $\mathbf{r}$ in the b-class, $\varphi(\mathbf{r})=\varphi(\mu \mathbf{r})$. Let $\mathbf{r}$ in the $\mathbf{b}$-class be such that $\mathbf{r} \neq \mu \mathbf{r}$. Then, since $\pi_{i}(\mathbf{r})<1$ for every $i \in X$, there exists a quasi-atom $\mathbf{s}$ in the $\mathbf{0}$-class such that $\mathbf{r} \leq \mathbf{s}^{*}$. It is sufficient therefore to show that, for each quasi-atom $\mathbf{s}$ in the $\mathbf{0}$-class, $\varphi\left(\mathbf{s}^{*}\right)=\varphi\left(\mu \mathbf{s}^{*}\right)$. Suppose that for some such $\mathbf{s}$, $\varphi\left(\mathbf{s}^{*}\right) \neq \varphi\left(\mu \mathbf{s}^{*}\right)$. For every quasi-atom $\mathbf{t} \leq \mathbf{s}^{*}$ that belongs to the $\mathbf{0}$-class, we now know that $\varphi(\mathbf{t})=\varphi(\mu \mathbf{t})$. Hence, by Lemma 4.1, either $\varphi(\mathbf{t})=0$ or else $\varphi(\mathbf{t})=a$ and $\varphi\left(\mathbf{s}^{*}\right) \in C_{m}(a)$. Since there are distinct such quasi-atoms which meet to $\mathbf{0}$, we may assume that $\varphi(\mathbf{t})=0$ for some such $\mathbf{t}$. Thus, 
$\varphi\left(\mathbf{t}^{*}\right)=1$ and, so, $\varphi\left(\mathbf{t}^{*}\right)=\varphi\left(\mu \mathbf{t}^{*}\right)$, which implies that $\varphi(\mathbf{b})=\varphi(\mu \mathbf{b})$. By Lemma 4.1, either $\varphi(\mathbf{b})=0$ or else $\varphi(\mathbf{b})=a$ and $\varphi\left(\mathbf{s}^{*}\right) \in C_{m}(a)$. However, $\mathbf{s}^{*}=\mathbf{b} \vee \bigvee\left\{\mathbf{t}: \mathbf{t}\right.$ is a quasi-atom in the $\mathbf{0}$-class and $\left.\mathbf{t} \leq \mathbf{s}^{*}\right\}$. Hence, either $\varphi\left(\mathbf{s}^{*}\right)=0$ or else $\varphi\left(\mathbf{s}^{*}\right)=a$ and $\varphi\left(\mathbf{s}^{*}\right) \in C_{m}(a)$, each of which is absurd.

5. Verification of $(\mathbf{P} 1)-(\mathbf{P} 4)$. We can now prove Theorem 1.1 by verifying that the family $\left(S_{W}: W \in P_{\text {fin }}(\omega)\right)$ satisfies the postulates (P1)(P4) of Proposition 2.1.

Since (P1) and (P2) clearly hold, we need only establish (P3) to conclude the following.

Proposition 5.1. The family $\left(S_{W}: W \in P_{\text {fin }}(\omega)\right)$ of finite pseudocomplemented semilattices satisfies the postulates $(\mathrm{P} 1)-(\mathrm{P} 3)$.

Proof. Let $X, Y \in P_{\text {fin }}(\omega)$ be such that $X \neq \emptyset$ and $S_{X} \in Q\left(S_{Y}\right)$. There is an embedding $\varphi: S_{X} \rightarrow\left(S_{Y}\right)^{I}$ for some finite set $I$. For each $j \in X$, $\varphi(\mathbf{e}) \neq \varphi\left(\nu_{j} \mathbf{e}\right)$ and hence there exists $i \in I$ such that the projection map $\pi_{i}:\left(S_{Y}\right)^{I} \rightarrow S_{Y}$ satisfies $\pi_{i} \varphi(\mathbf{e}) \neq \pi_{i} \varphi\left(\nu_{j} \mathbf{e}\right)$. Proposition 4.5 (applied to $\left.\pi_{i} \varphi: S_{X} \rightarrow S_{Y}\right)$ yields $j \in Y$ and $Y \subseteq X$. Since this holds for all $j \in X$, we have $Y=X$.

Lemma 5.2. If $X \in P_{\text {fin }}(\omega), \emptyset \neq Y \subseteq X$, and $\Theta$ is a congruence relation on $S_{X}$ with $\Gamma_{X} \leq \Theta$, then $S_{Y}$ is embeddable into $S_{X} /\left(\Theta \wedge \operatorname{Ker}\left(\pi_{Y}\right)\right)$, where $\Gamma_{X}$ denotes the Glivenko congruence on $S_{X}, \pi_{Y}$ denotes the projection map from $S_{X}$ onto $S_{Y}$, and $\operatorname{Ker}\left(\pi_{Y}\right)$ denotes the congruence kernel of the homomorphism $\pi_{Y}$.

Pro of. Let $\Psi$ denote the congruence relation on $S_{X}$ where, for $\mathbf{r}, \mathbf{s} \in S_{X}$, $\mathbf{r} \equiv \mathbf{s}(\Psi)$ if and only if $\pi_{Y}(\mathbf{r}) \equiv \pi_{Y}(\mathbf{s})\left(\Gamma_{Y}\right)$ where $\Gamma_{Y}$ denotes the Glivenko congruence on $S_{Y}$. We shall need the following:

LEMma 5.3. $\Psi=\Gamma_{X} \circ \operatorname{Ker}\left(\pi_{Y}\right)$.

Proof. Since $\Gamma_{X} \leq \Psi$ and $\operatorname{Ker}\left(\pi_{Y}\right) \leq \Psi, \Gamma_{X} \circ \operatorname{Ker}\left(\pi_{Y}\right) \leq \Psi$. To see that $\Psi \leq \Gamma_{X} \circ \operatorname{Ker}\left(\pi_{Y}\right)$, suppose $\mathbf{r}, \mathbf{s} \in S_{X}$ and $\mathbf{r} \equiv \mathbf{s}(\Psi)$. It must be shown that there exists $\mathbf{t} \in S_{X}$ such that $\mathbf{r} \Gamma_{X} \mathbf{t}$ and $\mathbf{t} \operatorname{Ker}\left(\pi_{Y}\right) \mathbf{s}$.

Clearly, for any such $\mathbf{t}$, it must be the case that $\pi_{j}(\mathbf{t})=\pi_{j}(\mathbf{s})$ for $j \in Y$. Thus, to determine a suitable $\mathbf{t}$, it is necessary to define $\pi_{i}(\mathbf{t})$ for $i \in X \backslash Y$. There are several cases depending on whether $\mathbf{r}$ and $\mathbf{s}$ belong to the $\mathbf{b}$-class, $\mu \mathbf{b}$-class, or $\mathbf{0}$-class of $S_{X}$ :

Let $\mathbf{r}$ belong to the $\mathbf{b}$-class. If $\mathbf{s}$ belongs to the $\mathbf{b}$-class, then set $\pi_{i}(\mathbf{t})=$ $\pi_{i}(\mathbf{r})$. When $\mathbf{s}$ belongs to the $\mu \mathbf{b}$-class, then let $\pi_{i}(\mathbf{t})=\mu \pi_{i}(\mathbf{r})$. For $\mathbf{s}$ to belong to the $\mathbf{0}$-class is not possible as $\pi_{Y}(\mathbf{r}) \equiv \pi_{Y}(\mathbf{s})\left(\Gamma_{Y}\right)$.

Let $\mathbf{r}$ belong to the $\mu \mathbf{b}$-class. If $\mathbf{s}$ belongs to the $\mathbf{b}$-class, then set $\pi_{i}(\mathbf{t})=$ $\left(\pi_{i}(\mathbf{r})\right)^{* *}$. If $\mathbf{s}$ belongs to the $\mu \mathbf{b}$-class, then let $\pi_{i}(\mathbf{t})=\pi_{i}(\mathbf{r})$. As above, it is not possible for $\mathbf{s}$ to belong to the $\mathbf{0}$-class. 
Let $\mathbf{r}$ belong to the $\mathbf{0}$-class. As above, the only possibility is that $\mathbf{s}$ also belongs to the $\mathbf{0}$-class. In which case $\pi_{i}(\mathbf{t})=\pi_{i}(\mathbf{r})$ will suffice.

Proof of Lemma 5.2 continued. Clearly, the set

$$
\left\{\left([\mathbf{r}] \Theta,[\mathbf{r}] \operatorname{Ker}\left(\pi_{Y}\right)\right): \mathbf{r} \in S_{X}\right\}
$$

is a subalgebra of $S_{X} / \Theta \times S_{X} / \operatorname{Ker}\left(\pi_{Y}\right)$. Denote this $p$-subsemilattice by $S$. Since $S_{X} /\left(\Theta \wedge \operatorname{Ker}\left(\pi_{Y}\right)\right)$ is isomorphic to $S$ via the isomorphism

$$
[\mathbf{r}]\left(\Theta \wedge \operatorname{Ker}\left(\pi_{Y}\right)\right) \mapsto\left([\mathbf{r}] \Theta,[\mathbf{r}] \operatorname{Ker}\left(\pi_{Y}\right)\right),
$$

to complete the proof of Lemma 5.2 it suffices to show that $S_{Y}$ is embeddable into $S$.

Define $\varphi: S_{Y} \rightarrow S$ where, for $\mathbf{s} \in S_{Y}, \varphi(\mathbf{s})=\left([\mathbf{r}] \Theta,[\mathbf{r}] \operatorname{Ker}\left(\pi_{Y}\right)\right)$ is such that $\mathbf{x} \Gamma_{X} \mathbf{r}$ and $\mathbf{r} \operatorname{Ker}\left(\pi_{Y}\right) \mathbf{x}^{\prime}$ for suitably defined $\mathbf{x}$ and $\mathbf{x}^{\prime} \in S_{X}$. The existence of such an $\mathbf{r}$ is guaranteed by Lemma 5.3 providing $\mathbf{x} \Psi \mathbf{x}^{\prime}$. The choice of $\mathbf{x}$ and $\mathbf{x}^{\prime}$ depends on whether $\mathbf{s}$ belongs to the $\mathbf{b}$-class, $\mu \mathbf{b}$-class, or 0-class of $S_{Y}$. There are three cases:

Let $\mathbf{s}$ belong to the $\mathbf{b}$-class of $S_{Y}$. Then $\mathbf{x}, \mathbf{x}^{\prime} \in S_{X}$, where, for $i \in Y$, $\pi_{i}(\mathbf{x})=\pi_{i}(\mathbf{s})^{* *}$ and $\pi_{i}\left(\mathbf{x}^{\prime}\right)=\pi_{i}(\mathbf{s})$ and, for $i \in X \backslash Y, \pi_{i}(\mathbf{x})=1$ and $\pi_{i}\left(\mathbf{x}^{\prime}\right)=1$.

Let $\mathbf{s}$ belong to the $\mu \mathbf{b}$-class of $S_{Y}$. Since $\mathbf{s}^{* *}$ belongs to the $\mathbf{b}$-class, $\mathbf{x}, \mathbf{x}^{\prime} \in S_{X}$ where, for $i \in Y, \pi_{i}(\mathbf{x})=\pi_{i}(\mathbf{s})^{* *}$ and $\pi_{i}\left(\mathbf{x}^{\prime}\right)=\pi_{i}(\mathbf{s})$ and, for $i \in X \backslash Y, \pi_{i}(\mathbf{x})=1$ and $\pi_{i}\left(\mathbf{x}^{\prime}\right)=\mu b$.

Let $\mathbf{s}$ belong to the $\mathbf{0}$-class of $S_{Y}$. Since $\mathbf{s}^{* *}$ also belongs to the $\mathbf{0}$-class, $\mathbf{x}, \mathbf{x}^{\prime} \in S_{X}$ where, for $i \in Y, \pi_{i}(\mathbf{x})=\pi_{i}(\mathbf{s})^{* *}$ and $\pi_{i}\left(\mathbf{x}^{\prime}\right)=\pi_{i}(\mathbf{s})$ and, for $i \in X \backslash Y, \pi_{i}(\mathbf{x})=0$ and $\pi_{i}\left(\mathbf{x}^{\prime}\right)=0$.

In each case $\mathbf{x} \Psi \mathbf{x}^{\prime}$. Thus, since $\Gamma_{X} \leq \Theta, \varphi$ is well-defined. It remains to show that $\varphi$ is a one-to-one homomorphism.

To see that $\varphi$ is one-to-one, suppose $\varphi(\mathbf{s})=\varphi(\mathbf{t})$ for some $\mathbf{s}, \mathbf{t} \in S_{Y}$. Let $\varphi(\mathbf{s})=\left([\mathbf{r}] \Theta,[\mathbf{r}] \operatorname{Ker}\left(\pi_{Y}\right)\right)$ and $\varphi(\mathbf{t})=\left([\mathbf{u}] \Theta,[\mathbf{u}] \operatorname{Ker}\left(\pi_{Y}\right)\right)$. Thus, $[\mathbf{r}] \operatorname{Ker}\left(\pi_{Y}\right)$ $=[\mathbf{u}] \operatorname{Ker}\left(\pi_{Y}\right)$. In particular, $\pi_{Y}(\mathbf{r})=\pi_{Y}(\mathbf{u})$. However, by the definition of $\varphi, \pi_{Y}(\mathbf{r})=\pi_{Y}(\mathbf{s})$ and $\pi_{Y}(\mathbf{u})=\pi_{Y}(\mathbf{t})$. Hence, $\mathbf{s}=\mathbf{t}$ as required.

To show that $\varphi$ is $\wedge$-preserving, we must establish that, for $\mathbf{s}, \mathbf{t} \in S_{Y}$, $\varphi(\mathbf{s} \wedge \mathbf{t})=\varphi(\mathbf{s}) \wedge \varphi(\mathbf{t})$. Let $\varphi(\mathbf{s} \wedge \mathbf{t})=\left([\mathbf{r}] \Theta,[\mathbf{r}] \operatorname{Ker}\left(\pi_{Y}\right)\right), \varphi(\mathbf{s})=$ $\left([\mathbf{u}] \Theta,[\mathbf{u}] \operatorname{Ker}\left(\pi_{Y}\right)\right)$, and $\varphi(\mathbf{t})=\left([\mathbf{v}] \Theta,[\mathbf{v}] \operatorname{Ker}\left(\pi_{Y}\right)\right)$, where $\mathbf{x}, \mathbf{x}^{\prime}, \mathbf{y}, \mathbf{y}^{\prime}, \mathbf{z}, \mathbf{z}^{\prime} \in$ $S_{X}$ determine appropriate $\mathbf{r}, \mathbf{u}, \mathbf{v} \in S_{X}$, respectively. Since $\Gamma_{X} \leq \Theta$, it is sufficient to show that $\mathbf{r} \Gamma_{X} \mathbf{u} \wedge \mathbf{v}$ and $\mathbf{r} \operatorname{Ker}\left(\pi_{Y}\right) \mathbf{u} \wedge \mathbf{v}$ in order to conclude that $\varphi(\mathbf{s} \wedge \mathbf{t})=\varphi(\mathbf{s}) \wedge \varphi(\mathbf{t})$. There are several cases depending on whether $\mathbf{s}$ and $\mathbf{t}$ belong to the $\mathbf{b}$-class, $\mu \mathbf{b}$-class, or $\mathbf{0}$-class of $S_{Y}$.

Let $\mathbf{s}$ belong to the $\mathbf{b}$-class of $S_{Y}$.

If $\mathbf{t}$ belongs to the $\mathbf{b}$-class, then so does $\mathbf{s} \wedge \mathbf{t}$. Thus, for $i \in Y, \pi_{i}(\mathbf{x})=$ $\pi_{i}(\mathbf{s} \wedge \mathbf{t})^{* *}, \pi_{i}(\mathbf{y})=\pi_{i}(\mathbf{s})^{* *}$, and $\pi_{i}(\mathbf{z})=\pi_{i}(\mathbf{t})^{* *}$ and, for $i \in X \backslash Y, \pi_{i}(\mathbf{x})=$ $\pi_{i}(\mathbf{y})=\pi_{i}(\mathbf{z})=1$. Since $(\mathbf{s} \wedge \mathbf{t})^{* *}=\mathbf{s}^{* *} \wedge \mathbf{t}^{* *}, \mathbf{x}=\mathbf{y} \wedge \mathbf{z}$. Consequently, as 
$\mathbf{x} \Gamma_{X} \mathbf{r}, \mathbf{y} \Gamma_{X} \mathbf{u}$, and $\mathbf{z} \Gamma_{X} \mathbf{v}$, it follows that $\mathbf{r} \Gamma_{X} \mathbf{u} \wedge \mathbf{v}$. Similarly, since in this case $\mathbf{x}=\mathbf{x}^{\prime}, \mathbf{y}=\mathbf{y}^{\prime}$, and $\mathbf{z}=\mathbf{z}^{\prime}$, we have $\mathbf{r} \operatorname{Ker}\left(\pi_{Y}\right) \mathbf{u} \wedge \mathbf{v}$.

If $\mathbf{t}$ belongs to the $\mu \mathbf{b}$-class, then so does $\mathbf{s} \wedge \mathbf{t}$. Thus, for $i \in Y, \pi_{i}(\mathbf{x})=$ $\pi_{i}(\mathbf{s} \wedge \mathbf{t})^{* *}, \pi_{i}(\mathbf{y})=\pi_{i}(\mathbf{s})^{* *}$, and $\pi_{i}(\mathbf{z})=\pi_{i}(\mathbf{t})^{* *}$ and, for $i \in X \backslash Y, \pi_{i}(\mathbf{x})=$ $\pi_{i}(\mathbf{y})=\pi_{i}(\mathbf{z})=1$. As before, it follows that $\mathbf{r} \Gamma_{X} \mathbf{u} \wedge \mathbf{v}$. For $i \in Y, \pi_{i}\left(\mathbf{x}^{\prime}\right)=$ $\pi_{i}(\mathbf{s} \wedge \mathbf{t}), \pi_{i}\left(\mathbf{y}^{\prime}\right)=\pi_{i}(\mathbf{s})$, and $\pi_{i}\left(\mathbf{z}^{\prime}\right)=\pi_{i}(\mathbf{t})$ and, for $i \in X \backslash Y, \pi_{i}\left(\mathbf{x}^{\prime}\right)=\mu b$, $\pi_{i}\left(\mathbf{y}^{\prime}\right)=1, \pi_{i}\left(\mathbf{z}^{\prime}\right)=\mu b$. In particular, $\mathbf{x}^{\prime}=\mathbf{y}^{\prime} \wedge \mathbf{z}^{\prime}$ and, as $\mathbf{x}^{\prime} \operatorname{Ker}\left(\pi_{Y}\right) \mathbf{r}$, $\mathbf{y}^{\prime} \operatorname{Ker}\left(\pi_{Y}\right) \mathbf{u}$, and $\mathbf{z}^{\prime} \operatorname{Ker}\left(\pi_{Y}\right) \mathbf{v}$, it follows that $\mathbf{r} \operatorname{Ker}\left(\pi_{Y}\right) \mathbf{u} \wedge \mathbf{v}$.

If $\mathbf{t}$ belongs to the $\mathbf{0}$-class, then so does $\mathbf{s} \wedge \mathbf{t}$. Hence, for $i \in Y, \pi_{i}(\mathbf{x})=$ $\pi_{i}(\mathbf{s} \wedge \mathbf{t})^{* *}, \pi_{i}(\mathbf{y})=\pi_{i}(\mathbf{s})^{* *}$, and $\pi_{i}(\mathbf{z})=\pi_{i}(\mathbf{t})^{* *}$ and, for $i \in X \backslash Y, \pi_{i}(\mathbf{x})=0$, $\pi_{i}(\mathbf{y})=1$, and $\pi_{i}(\mathbf{z})=0$. As above, $\mathbf{r} \Gamma_{X} \mathbf{u} \wedge \mathbf{v}$. For $i \in Y, \pi_{i}\left(\mathbf{x}^{\prime}\right)=\pi_{i}(\mathbf{s} \wedge \mathbf{t})$, $\pi_{i}\left(\mathbf{y}^{\prime}\right)=\pi_{i}(\mathbf{s})$, and $\pi_{i}\left(\mathbf{z}^{\prime}\right)=\pi_{i}(\mathbf{t})$ and, for $i \in X \backslash Y, \pi_{i}\left(\mathbf{x}^{\prime}\right)=0, \pi_{i}\left(\mathbf{y}^{\prime}\right)=1$, and $\pi_{i}\left(\mathbf{z}^{\prime}\right)=0$. Once more, $\mathbf{r} \operatorname{Ker}\left(\pi_{Y}\right) \mathbf{u} \wedge \mathbf{v}$.

Let $\mathbf{s}$ belong to the $\mu \mathbf{b}$-class of $S_{Y}$.

Since $\wedge$ is commutative, it is now no longer necessary to consider $\mathbf{t}$ a member of the b-class.

If $\mathbf{t}$ belongs to the $\mu \mathbf{b}$-class of $S_{Y}$, then so does $\mathbf{s} \wedge \mathbf{t}$. Consequently, for $i \in Y, \pi_{i}(\mathbf{x})=\pi_{i}(\mathbf{s} \wedge \mathbf{t})^{* *}, \pi_{i}(\mathbf{y})=\pi_{i}(\mathbf{s})^{* *}$, and $\pi_{i}(\mathbf{z})=\pi_{i}(\mathbf{t})^{* *}$ and, for $i \in X \backslash Y, \pi_{i}(\mathbf{x})=\pi_{i}(\mathbf{y})=\pi_{i}(\mathbf{z})=1$. For $i \in Y, \pi_{i}\left(\mathbf{x}^{\prime}\right)=\pi_{i}(\mathbf{s} \wedge \mathbf{t}), \pi_{i}\left(\mathbf{y}^{\prime}\right)=$ $\pi_{i}(\mathbf{s})$, and $\pi_{i}\left(\mathbf{z}^{\prime}\right)=\pi_{i}(\mathbf{t})$ and, for $i \in X \backslash Y, \pi_{i}\left(\mathbf{x}^{\prime}\right)=\pi_{i}\left(\mathbf{y}^{\prime}\right)=\pi_{i}\left(\mathbf{z}^{\prime}\right)=\mu b$. In particular, $\mathbf{x}=\mathbf{y} \wedge \mathbf{z}$ and $\mathbf{x}^{\prime}=\mathbf{y}^{\prime} \wedge \mathbf{z}^{\prime}$. Hence $\mathbf{r} \Gamma_{X} \mathbf{u} \wedge \mathbf{v}$ and $\mathbf{r} \operatorname{Ker}\left(\pi_{Y}\right) \mathbf{u} \wedge \mathbf{v}$.

If $\mathbf{t}$ belongs to the $\mathbf{0}$-class, then so does $\mathbf{s} \wedge \mathbf{t}$. For $i \in Y, \pi_{i}(\mathbf{x})=\pi_{i}(\mathbf{s} \wedge \mathbf{t})^{* *}$, $\pi_{i}(\mathbf{y})=\pi_{i}(\mathbf{s})^{* *}, \pi_{i}(\mathbf{z})=\pi_{i}(\mathbf{t})^{* *}, \pi_{i}\left(\mathbf{x}^{\prime}\right)=\pi_{i}(\mathbf{s} \wedge \mathbf{t}), \pi_{i}\left(\mathbf{y}^{\prime}\right)=\pi_{i}(\mathbf{s})$, and $\pi_{i}\left(\mathbf{z}^{\prime}\right)=\pi_{i}(\mathbf{t})$ and, for $i \in X \backslash Y, \pi_{i}(\mathbf{x})=0, \pi_{i}(\mathbf{y})=1, \pi_{i}(\mathbf{z})=0, \pi_{i}\left(\mathbf{x}^{\prime}\right)=0$, $\pi_{i}\left(\mathbf{y}^{\prime}\right)=\mu b$, and $\pi_{i}\left(\mathbf{z}^{\prime}\right)=0$. Clearly, once more $\mathbf{x}=\mathbf{y} \wedge \mathbf{z}$ and $\mathbf{x}^{\prime}=\mathbf{y}^{\prime} \wedge \mathbf{z}^{\prime}$, as required.

Let $\mathbf{s}$ belong to the $\mathbf{0}$-class of $S_{Y}$.

By the commutativity of $\wedge$, it only remains to consider $\mathbf{t}$ a member of the $\mathbf{0}$-class. Obviously, $\mathbf{s} \wedge \mathbf{t}$ is also a member of the $\mathbf{0}$-class. For $i \in Y$, $\pi_{i}(\mathbf{x})=\pi_{i}(\mathbf{s} \wedge \mathbf{t})^{* *}, \pi_{i}(\mathbf{y})=\pi_{i}(\mathbf{s})^{* *}, \pi_{i}(\mathbf{z})=\pi_{i}(\mathbf{t})^{* *}, \pi_{i}\left(\mathbf{x}^{\prime}\right)=\pi_{i}(\mathbf{s} \wedge \mathbf{t})$, $\pi_{i}\left(\mathbf{y}^{\prime}\right)=\pi_{i}(\mathbf{s})$, and $\pi_{i}\left(\mathbf{z}^{\prime}\right)=\pi_{i}(\mathbf{t})$ and, for $i \in X \backslash Y, \pi_{i}(\mathbf{x})=\pi_{i}(\mathbf{y})=$ $\pi_{i}(\mathbf{z})=\pi_{i}\left(\mathbf{x}^{\prime}\right)=\pi_{i}\left(\mathbf{y}^{\prime}\right)=\pi_{i}\left(\mathbf{z}^{\prime}\right)=0$. Thus, in this case, as for every case, $\mathbf{r} \Gamma_{X} \mathbf{u} \wedge \mathbf{v}$ and $\mathbf{r} \operatorname{Ker}\left(\pi_{Y}\right) \mathbf{u} \wedge \mathbf{v}$. In particular, $\varphi$ is $\wedge$-preserving.

To see that $\varphi$ is *-preserving, it is necessary to establish that, for $\mathbf{s} \in S_{Y}, \varphi\left(\mathbf{s}^{*}\right)=\varphi(\mathbf{s})^{*}$. Let $\varphi\left(\mathbf{s}^{*}\right)=\left([\mathbf{r}] \Theta,[\mathbf{r}] \operatorname{Ker}\left(\pi_{Y}\right)\right)$ and $\varphi(\mathbf{s})=$ $\left([\mathbf{u}] \Theta,[\mathbf{u}] \operatorname{Ker}\left(\pi_{Y}\right)\right)$, where $\mathbf{r}$ and $\mathbf{u}$ are determined by $\mathbf{x}, \mathbf{x}^{\prime}, \mathbf{y}, \mathbf{y}^{\prime} \in S_{X}$. As above, it is sufficient to show that $\mathbf{r} \Gamma_{X} \mathbf{u}^{*}$ and $\mathbf{r} \operatorname{Ker}\left(\pi_{Y}\right) \mathbf{u}^{*}$.

If $\mathbf{s}$ is in the $\mathbf{b}$-class, then $\mathbf{s}^{*}$ is in the $\mathbf{0}$-class. Thus, for $i \in Y, \pi_{i}(\mathbf{x})=$ $\pi_{i}\left(\mathbf{s}^{*}\right)^{* *}$ and $\pi_{i}(\mathbf{y})=\pi_{i}(\mathbf{s})^{* *}$ and, for $i \in X \backslash Y, \pi_{i}(\mathbf{x})=0$ and $\pi_{i}(\mathbf{y})=1$. Since $\mathbf{x}=\mathbf{y}^{*}, \mathbf{x} \Gamma_{X} \mathbf{r}$, and $\mathbf{y} \Gamma_{X} \mathbf{u}$, it follows that $\mathbf{r} \Gamma_{X} \mathbf{u}^{*}$. For $i \in Y, \pi_{i}\left(\mathbf{x}^{\prime}\right)=$ $\pi_{i}\left(\mathbf{s}^{*}\right)$ and $\pi_{i}\left(\mathbf{y}^{\prime}\right)=\pi_{i}(\mathbf{s})$. Since $\mathbf{x}^{\prime} \operatorname{Ker}\left(\pi_{Y}\right) \mathbf{y}^{\prime *}$, we have $\mathbf{r} \operatorname{Ker}\left(\pi_{Y}\right) \mathbf{u}^{*}$. 
If $\mathbf{s}$ is in the $\mu \mathbf{b}$-class, then $\mathbf{s}^{*}$ is in the $\mathbf{0}$-class. Thus, for $i \in Y, \pi_{i}(\mathbf{x})=$ $\pi_{i}\left(\mathbf{s}^{*}\right)^{* *}$ and $\pi_{i}(\mathbf{y})=\pi_{i}(\mathbf{s})^{* *}$ and, for $i \in X \backslash Y, \pi_{i}(\mathbf{x})=0$ and $\pi_{i}(\mathbf{y})=1$. Since $\mathbf{x}=\mathbf{y}^{*}$, we have $\mathbf{r} \Gamma_{X} \mathbf{u}^{*}$. For $i \in Y, \pi_{i}\left(\mathbf{x}^{\prime}\right)=\pi_{i}\left(\mathbf{s}^{*}\right)$ and $\pi_{i}\left(\mathbf{y}^{\prime}\right)=\pi_{i}(\mathbf{s})$. Since $\mathbf{x}^{\prime} \operatorname{Ker}\left(\pi_{Y}\right) \mathbf{y}^{\prime *}$, we obtain $\mathbf{r} \operatorname{Ker}\left(\pi_{Y}\right) \mathbf{u}^{*}$.

If $\mathbf{s}$ is in the $\mathbf{0}$-class, then $\mathbf{s}^{*}$ is in the $\mathbf{b}$-class. Thus, for $i \in Y, \pi_{i}(\mathbf{x})=$ $\pi_{i}\left(\mathbf{s}^{*}\right)^{* *}$ and $\pi_{i}(\mathbf{y})=\pi_{i}(\mathbf{s})^{* *}$ and, for $i \in X \backslash Y, \pi_{i}(\mathbf{x})=1$ and $\pi_{i}(\mathbf{y})=0$. Hence, $\mathbf{x}=\mathbf{y}^{*}$ and, so, $\mathbf{r} \Gamma_{X} \mathbf{u}^{*}$. For $i \in Y, \pi_{i}\left(\mathbf{x}^{\prime}\right)=\pi_{i}\left(\mathbf{s}^{*}\right)$ and $\pi_{i}\left(\mathbf{y}^{\prime}\right)=$ $\pi_{i}(\mathbf{s})$. Hence, $\mathbf{x}^{\prime} \operatorname{Ker}\left(\pi_{Y}\right) \mathbf{y}^{\prime *}$ which implies $\mathbf{r} \operatorname{Ker}\left(\pi_{Y}\right) \mathbf{u}^{*}$. Consequently, $\varphi$ is $*$-preserving.

Proposition 5.4. The family $\left(S_{W}: W \in P_{\text {fin }}(\omega)\right)$ of finite pseudocomplemented semilattices satisfies the postulate $(\mathrm{P} 4)$.

Proof. Let $X \in P_{\text {fin }}(\omega)$ and $B, C \in Q\left(\left\{S_{W}: W \in P_{\text {fin }}(\omega)\right\}\right)$ be finite algebras such that $S_{X}$ is a subalgebra of $B \times C$. We must exhibit $Y, Z \in$ $P_{\text {fin }}(\omega)$ with $S_{Y} \in Q(B), S_{Z} \in Q(C)$, and $X=Y \cup Z$. Clearly, with no loss in generality, we may assume that either $B$ or $C$ is a non-trivial algebra.

Let $\pi_{B}$ and $\pi_{C}$ denote the projections of $S_{X}$ into $B$ and $C$, respectively. Define

$$
Y=\left\{i \in X: \pi_{B}(\mathbf{e}) \neq \pi_{B}\left(\nu_{i} \mathbf{e}\right)\right\}, \quad Z=\left\{i \in X: \pi_{C}(\mathbf{e}) \neq \pi_{C}\left(\nu_{i} \mathbf{e}\right)\right\} .
$$

Since $S_{X}$ is a subalgebra of $B \times C, X=Y \cup Z$. Observe, in passing, that $\operatorname{Ker}\left(\pi_{B}\right) \wedge \operatorname{Ker}\left(\pi_{C}\right)$ is the identity on $S_{X}$. Further, as $B \times C$ does not contain a trivial subalgebra, it follows that $X \neq \emptyset$ and, hence, either $Y \neq \emptyset$ or $Z \neq \emptyset$.

To begin with, suppose that either $Y=\emptyset$ or $Z=\emptyset$, say $Z=\emptyset$, and so $Y=X$. Since $S_{\emptyset} \in Q(C)$, it is sufficient to show that $S_{X}$ is embeddable in $B$, which implies $S_{Y}=S_{X} \in Q(B)$. Because $B \in Q\left(\left\{S_{W}: W \in P_{\text {fin }}(\omega)\right\}\right)$ and $Q\left(\left\{S_{W}: W \in P_{\text {fin }}(\omega)\right\}\right)=Q\left(\left\{S_{i}: i \in \omega\right\}\right)$, there exists a set $W \in P_{\text {fin }}(\omega)$ and an embedding $\varphi$ of $B$ in $\prod\left(S_{i}: i \in W\right)$. Thus,

$$
S_{X} \stackrel{\pi_{B}}{\hookrightarrow} B \stackrel{\varphi}{\longmapsto} \prod\left(S_{i}: i \in W\right) .
$$

Since $X=Y$, Proposition 4.3 implies that $X \subseteq W$ and, for each $i \in X$, the map $\pi_{i} \circ \varphi \circ \pi_{B}: S_{X} \rightarrow S_{i}$ satisfies $\operatorname{Ker}\left(\pi_{i} \circ \varphi \circ \pi_{B}\right)=\operatorname{Ker}\left(\pi_{i}^{\prime}\right)$ where $\pi_{i}$ refers to the projection from $\prod\left(S_{i}: i \in W\right)$ into $S_{i}$ and $\pi_{i}^{\prime}$ refers to the projection from $S_{X}$ into $S_{i}$. Let $\mathbf{r}, \mathbf{s} \in S_{X}$ and $\mathbf{r} \equiv \mathbf{s}\left(\operatorname{Ker}\left(\pi_{B}\right)\right)$. Thus, $\pi_{i} \circ \varphi \circ \pi_{B}(\mathbf{r})=\pi_{i} \circ \varphi \circ \pi_{B}(\mathbf{s})$ for all $i \in X$. Therefore $\pi_{i}(\mathbf{s})=\pi_{i}(\mathbf{r})$ for all $i \in X$, which means that $\mathbf{r}=\mathbf{s}$. It follows that $\operatorname{Ker}\left(\pi_{B}\right)$ is the identity on $S_{X}$, and so $S_{X}$ is embeddable in $B$. In particular, $S_{X} \in Q(B)$.

Suppose that both $Y \neq \emptyset$ and $Z \neq \emptyset$. As before, there exists a set $W$ and an embedding $\varphi$ of $B$ in $\prod\left(S_{i}: i \in W\right)$ and, likewise, there exists a set $V$ and an embedding $\psi$ of $C$ in $\prod\left(S_{i}: i \in V\right)$. It is sufficient to show that $S_{Y}$ embeds in $S_{X} / \operatorname{Ker}\left(\pi_{B}\right)$ and that $S_{Z}$ embeds in $S_{X} / \operatorname{Ker}\left(\pi_{C}\right)$ since this implies that $S_{Y} \in Q(B)$ and $S_{Z} \in Q(C)$. We only show that $S_{Y}$ embeds 
in $S_{X} / \operatorname{Ker}\left(\pi_{B}\right)$; the proof that $S_{Z}$ embeds in $S_{X} / \operatorname{Ker}\left(\pi_{C}\right)$ is similar. By Proposition 4.3, $Y \subseteq W$ and, for each $i \in Y$, the map $\pi_{i} \circ \varphi \circ \pi_{B}: S_{X} \rightarrow$ $S_{i}$ satisfies $\operatorname{Ker}\left(\pi_{i} \circ \varphi \circ \pi_{B}\right)=\operatorname{Ker}\left(\pi_{i}^{\prime}\right)$ where $\pi_{i}$ and $\pi_{i}^{\prime}$ are as above. In particular, $\bigwedge\left(\operatorname{Ker}\left(\pi_{i} \circ \varphi \circ \pi_{B}\right): i \in Y\right)=\operatorname{Ker}\left(\pi_{Y}\right)$ where $\pi_{Y}$ denotes the projection on $S_{X}$. Since $\bigwedge\left(\operatorname{Ker}\left(\pi_{i} \circ \varphi \circ \pi_{B}\right): i \in W\right)=\operatorname{Ker}\left(\pi_{B}\right)$, it follows that

$$
\operatorname{Ker}\left(\pi_{B}\right)=\bigwedge\left(\operatorname{Ker}\left(\pi_{i} \circ \varphi \circ \pi_{B}\right): i \in W \backslash Y\right) \wedge \operatorname{Ker}\left(\pi_{Y}\right) .
$$

However, from Proposition 4.3 it follows that, for each $i \in W \backslash Y, \pi_{i}$ 。 $\varphi \circ \pi_{B}(\mathbf{e})=\pi_{i} \circ \varphi \circ \pi_{B}\left(\nu_{j} \mathbf{e}\right)$ for all $j \in X$. Thus, by Proposition 4.6, $\Gamma_{X} \leq \operatorname{Ker}\left(\pi_{i} \circ \varphi \circ \pi_{B}\right)$ for each $i \in W \backslash Y$, and so $\Gamma_{X} \leq \bigwedge\left(\operatorname{Ker}\left(\pi_{i} \circ \varphi \circ \pi_{B}\right)\right.$ : $i \in W \backslash Y)$. By Lemma 5.2, $S_{Y}$ is embeddable in $S_{X} / \bigwedge\left(\operatorname{Ker}\left(\pi_{i} \circ \varphi \circ \pi_{B}\right): i \in\right.$ $W \backslash Y) \wedge \operatorname{Ker}\left(\pi_{Y}\right)$, which, by $(\dagger)$, implies $S_{Y}$ is embeddable in $S_{X} / \operatorname{Ker}\left(\pi_{B}\right)$, as required.

\section{References}

[1] M. E. Adams, Implicational classes of pseudocomplemented distributive lattices, J. London Math. Soc. 13 (1976), 381-384.

[2] M. E. Adams and W. Dziobiak, Q-universal quasivarieties of algebras, Proc. Amer. Math. Soc. 120 (1994), 1053-1059.

[3] -, - Lattices of quasivarieties of 3-element algebras, J. Algebra 166 (1994), 181210.

[4] M. E. Adams and M. Gould, A construction for pseudocomplemented semilattices and two applications, Proc. Amer. Math. Soc. 106 (1989), 899-905.

[5] S. Burris and H. P. Sankappanavar, A Course in Universal Algebra, Springer, New York, 1981.

[6] W. Dziobiak, On subquasivariety lattices of some varieties related with distributive p-algebras, Algebra Universalis 21 (1985), 62-67.

[7] G. Grätzer, General Lattice Theory, Birkhäuser, Basel, 1978.

[8] G. T. Jones, Pseudocomplemented semilattices, Ph.D. dissertation, U.C.L.A., 1972.

[9] A. I. Mal'cev, On certain frontier questions in algebra and mathematical logic, Proc. Internat. Congr. of Mathematicians, Moscow 1966, Mir, 1968, 217-231 (in Russian).

[10] —, Algebraic Systems, Grundlehren Math. Wiss. 192, Springer, New York, 1973.

[11] H. P. Sankappanavar, Remarks on subdirectly irreducible pseudocomplemented semi-lattices and distributive pseudocomplemented lattices, Math. Japon. 25 (1980), 519-521.

[12] M. V. Sapir, The lattice of quasivarieties of semigroups, Algebra Universalis 21 (1985), 172-180.

[13] J. Schmid, Lee classes and sentences for pseudocomplemented semilattices, ibid. 25 (1988), 223-232.

[14] - On amalgamation classes of pseudocomplemented semilattices, ibid. 29 (1992), 402-418.

[15] A. Shafaat, On implicational completeness, Canad. J. Math. 26 (1974), 761-768. 
[16] M. P. Tropin, An embedding of a free lattice into the lattice of quasivarieties of distributive lattices with pseudocomplementation, Algebra i Logika 22 (1983), 159167 (in Russian).

[17] A. Wroński, The number of quasivarieties of distributive lattices with pseudocomplementation, Polish Acad. Sci. Inst. Philos. Sociol. Sect. Logic 5 (1976), 115-121.

DEPARTMENT OF MATHEMATICS STATE UNIVERSITY OF NEW YORK NEW PALTZ, NEW YORK 12561

U.S.A.

E-mail: ADAMSM@MATRIX.NEWPALTZ.EDU

DEPARTMENT OF MATHEMATICS

VANDERBILT UNIVERSITY

NASHVILLE, TENNESSEE 37240

U.S.A.

E-mail: GOULDM00@VUCTRVX1.BITNET

Current address of W. Dziobiak:

DEPARTMENT OF MATHEMATICS

UNIVERSITY OF PUERTO RICO

MAYAGUEZ, PUERTO RICO 00681-5000

U.S.A.

E-mail: W_DZIOBIAK\%RUMAC@UPR1.UPR.CLU.EDU
INSTITUTE OF MATHEMATICS NICHOLAS COPERNICUS UNIVERSITY

CHOPINA $12 / 18$

87-100 TORUŃ, POLAND

E-mail: DZIOBIAK@PLTUMK11.BITNET

INSTITUTE OF MATHEMATICS UNIVERSITY OF BERN

CH-3012 BERN

SWITZERLAND

E-mail: SCHMID@MATH-STAT.UNIBE.CH

Received 28 February 1994 